\title{
(An)isotropy of the Hubble diagram: comparing hemispheres
}

\author{
D. J. Schwarz and B. Weinhorst
}

Fakultät für Physik, Postfach 100131, Universität Bielefeld, 33501 Bielefeld, Germany

e-mail: [dschwarz; bwein]@physik .uni-bielefeld.de

Received 1 June 2007 / Accepted 28 August 2007

\begin{abstract}
Aims. We test the isotropy of the Hubble diagram. At small redshifts, this is possible without assumptions on the cosmic inventory and provides a fundamental test of the cosmological principle. At higher redshift we check for the self-consistency of the $\Lambda \mathrm{CDM}$ model.

Methods. At small redshifts, we use public supernovae ( $\mathrm{SNe}$ ) Ia data to determine the deceleration parameter $q_{0}$ and the $\mathrm{SN}$ calibration on opposite hemispheres. For the complete data sets we fit $\Omega_{\mathrm{M}}$ and the $\mathrm{SN}$ calibration on opposite hemispheres.

Results. A statistically significant anisotropy of the Hubble diagram at redshifts $z<0.2$ is discovered (>95\%C.L.). While data from the North Galactic hemisphere favour the accelerated expansion of the Universe, data from the South Galactic hemisphere are not conclusive. The hemispheric asymmetry is maximal toward a direction close to the equatorial poles. The discrepancy between the equatorial North and South hemispheres shows up in the SN calibration. For the $\Lambda$ CDM model fitted to all available SNe, we find the same asymmetry.

Conclusions. The alignment of discrepancies between hemispheric Hubble diagrams with the equatorial frame seems to point toward a systematic error in the $\mathrm{SN}$ search, observation, analysis or data reduction. We also find that our model independent test cannot exclude the case of the deceleration of the expansion at a statistically significant level.
\end{abstract}

Key words. cosmology: observations - large-scale structure of Universe - supernovae: general

\section{Introduction}

The Hubble law is a direct consequence of the cosmological principle. Modern Hubble diagrams from supernovae Ia (SNe Ia) confirm the Hubble law and provide evidence for an accelerated expansion of the Universe (Riess et al. 1998; Perlmutter et al. 1999). In these studies the isotropy of the Hubble diagram is assumed. The purpose of this work is to provide quantitative tests of the isotropy of SNe Ia Hubble diagrams, beyond the identification of the cosmic microwave dipole in the local $\mathrm{SNe}$ Ia data (Riess et al. 1995).

A test of the isotropy of Hubble diagrams is interesting for at least three reasons: 1. for checking the validity of the cosmological principle (Kolatt \& Lahav 2001; McClure \& Dyer 2007), 2. to measure expected deviations from the isotropy (due to Local Group motion, peculiar velocities, and other effects from structure formation (Sasaki 1987; Radburn-Smith et al. 2004; Bonvin et al. 2006a; Hui \& Greene 2006; Weinhorst 2006; Cooray \& Caldwell 2006; Haugbølle et al. 2006; Neill et al. 2007; Wang 2007; Hannestad et al. 2007; Gordon et al. 2007)), 3. to search for systematic errors in the observations and their analysis (Kolatt \& Lahav 2001; Gupta et al. 2007).

The cosmological principle states that the statistical distribution of matter and light in the Universe is isotropic and homogeneous in space. The principle itself may either be motivated by the idea of cosmological inflation or by a simplicity argument. As a consequence of the ergodic theorem (spatial averaging replaces ensemble averaging) the spatial isotropy and homogeneity of the statistical distributions becomes an approximate symmetry of the space-time metric and matter distribution at large scales.
Consequently, the cosmological principle implies that distances, angles, time intervals etc. at large scales can be measured according to the ruler sticks and clocks described by the Robertson-Walker line element. The redshift of light from distant (with the expansion comoving) objects and the Hubble law for redshift $z \ll 1$ follow directly (without making use of Einstein's equation), i.e. the Hubble law does not depend on the details of the cosmological model (like the inventory of the Universe). The Hubble diagram (distance or magnitude versus redshift) therefore provides one of the most fundamental tests of modern cosmology. It provides a test of the cosmological principle and the idea that the space-time is correctly modelled as a riemannian manifold.

However, the local Universe is neither isotropic nor homogeneous. Consequently, the observations are expected to approach the Hubble law at some $z>0$ only. Deviations from the isotropic and homogeneous Universe arise from structure formation and can be described by linear perturbation theory at large scales. Thus in order to measure the Hubble constant, the deceleration parameter and other cosmological parameters of an isotropic and homogenous model, it is compulsory to demonstrate that the effects from structure formation are either negligible or properly considered in the error bars.

Most importantly, one would like to firmly establish the acceleration of the Universe in a model independent way; i.e. to measure the kinematics of the Universe without assuming the validity of Einstein's equation and without assumptions on the matter content of the Universe. This leads to a dilemma: at very small redshifts, the Hubble law does not hold, since the local Universe is inhomogeneous. At high redshift, $z \sim 1$, the luminosity distance $d_{\mathrm{L}}$ (or equivalently the distance modulus $m-M$ ) depends on the detailed cosmological model. Therefore, such an 
analysis must be restricted to a finite range of redshifts below one, but must exclude the most local SNe.

A simple and powerful method to establish the existence of such a range of redshifts is to test the isotropy of the Hubble diagram. To be more precise, the isotropy of the Hubble diagram is a necessary, but not a sufficient condition for the existence of such an interval. Here we use SNe Ia data to test the isotropy of the Hubble diagram on opposite hemispheres of the sky.

This test is also closely related to the issue of fairly sampling the Universe with SNe. In other words, is it suitable to determine the global Hubble rate from a local measurement. The 1998 cosmology-revolution (Riess et al. 1998; Perlmutter et al. 1999) relies on the assumption that the $\mathrm{SNe}$ Ia at low redshifts (say $z<0.2$ ) represent a fair sample of the Universe and that the Hubble law is a good approximation to the data. Based on $\mathrm{SNe}$ Ia at low and high redshifts together, it has been concluded that the present expansion of the Universe accelerates.

At the same time, our test provides a cross check for the measurements of the Hubble constant $H_{0}$ and the deceleration parameter $q_{0}$. The most commonly adopted values for the inflationary $\Lambda$ cold dark matter model $(\Lambda \mathrm{CDM})$ are from the HST key project $H_{0}=72 \pm 8 \mathrm{~km} \mathrm{~s}^{-1} \mathrm{Mpc}^{-1}$ (Freedman et al. 2001) and from a fit to WMAP data $H_{0}=73_{-4}^{+3} \mathrm{~km} \mathrm{~s}^{-1} \mathrm{Mpc}^{-1}$ and $q_{0}=\left(3 \Omega_{\mathrm{M}}-2\right) / 2=-0.64_{-0.06}^{+0.04}$ (derived, not directly measured) (Spergel et al. 2006). The calibration of SNe Ia used in the HST key project analysis has recently been criticised by Sandage et al. (2006). The HST key project analysis is based on a set of cepheides from a metal-poor environment, whereas most of the $\mathrm{SNe}$ of the sample are in metal-rich galaxies. Sandage et al. (2006) obtain $H_{0}=62.3 \pm 1.5 \pm 5.0 \mathrm{~km} \mathrm{~s}^{-1} \mathrm{Mpc}^{-1}$, using cepheides from a metal-rich environment and a significantly larger number of calibrators and $\mathrm{SNe}$. At face value, there is now some tension between $\mathrm{CMB}$ measurements and the direct determination from the Hubble diagram. A possible explanation would be that the local value does not coincide with the global one. Our test is suited to detect such a situation, as we would expect it to go along with an anisotropy of the Hubble diagram.

In the second part of this work we present a model dependent analysis of the isotropy of Hubble diagrams, relying on the spatially flat $\Lambda \mathrm{CDM}$ model, i.e. a model with two free parameters $H_{0}$ and $\Omega_{\mathrm{M}}$.

The paper is structured as follows. In Sect. 2 we comment on some basic properties of the Hubble law. In Sect. 3 we describe the four SNe Ia data sets that we are using. The method of our tests is explained in Sects. 4 and 5 contains the results. We conclude in Sect. 6.

\section{Hubble law}

The luminosity distance $d_{\mathrm{L}}$ is a function of redshift and angular position of an observed object. It is linked to the distance modulus $m-M=5 \log \left(d_{\mathrm{L}} / 1 \mathrm{Mpc}\right)+25$. The observed redshift $z$ of the object contains information on its peculiar velocity, the peculiar velocity of the observer and the cosmological redshift. If we assume that the cosmological principle holds and that the observed objects are comoving with the expansion of the Universe, one finds the Hubble law

$$
\begin{aligned}
d_{\mathrm{L}}(z)= & \frac{c}{H_{0}}\left[z+\left(1-q_{0}\right) \frac{z^{2}}{2}\right. \\
& \left.+\left(-j_{0}+3 q_{0}^{2}+q_{0}-1-\frac{k}{a_{0}^{2}} \frac{c^{2}}{H_{0}^{2}}\right) \frac{z^{3}}{6}+O\left(z^{4}\right)\right],
\end{aligned}
$$

where $j$ denotes the jerk, $k=0, \pm 1$ the normalised, comoving curvature and $a$ the scale factor. In terms of the scale factor and its derivatives

$H=\frac{\dot{a}}{a}, \quad q=-\frac{\ddot{a}}{a} \frac{1}{H^{2}}, \quad j=\frac{\dot{a}}{a} \frac{1}{H^{3}}$.

We see that the luminosity distance does not depend on the geometry of the Universe up to order $z^{2}$, while the terms of order $z^{3}$ depend on jerk and geometry. We restrict our analysis to a test of the Hubble law up to order $z^{2}$. A useful reference is the emptyUniverse model, which is characterised by $q=j=0$. Using the Einstein equation for the reference model we find additionally $H^{2} / c^{2}=-k / a^{2}$, thus $k=-1$ and $H_{0} d_{\mathrm{L}}(z)=c z(1+z / 2)$.

Let us estimate the error from neglecting jerk and curvature in (1). For a general $\Lambda$ CDM model, we find $q_{0}=\left(\Omega_{M}-2 \Omega_{\Lambda}\right) / 2$ and $j_{0}=\Omega_{\mathrm{M}}+\Omega_{\Lambda}$. In the special case of the Einstein-de Sitter model $\left(q_{0}=1 / 2, j_{0}=1, k=0\right)$, the ratio of the third order term to the second order term in (1) becomes $-1 / 2$ and thus the error from neglecting the third order term amounts to $10 \%$ at $z=0.2$. For the WMAP best-fit flat $\Lambda \mathrm{CDM}$ model the error at the same redshift is $6 \%$. Therefore, in order to obtain $q_{0}$ and its confidence contours at $10 \%$ theoretical accuracy or better, we must restrict the model independent fits to $\mathrm{SNe}$ at redshifts below $z=0.2$.

For the spatially flat $\Lambda \mathrm{CDM}$ model (assuming Einstein's equation) the exact expression for the luminosity distance is given by

$d_{\mathrm{L}}(z)=c(1+z) \int_{0}^{z} \frac{\mathrm{d} z^{\prime}}{H\left(z^{\prime}\right)}$

with $H(z)^{2}=H_{0}^{2}\left[\Omega_{\mathrm{M}}(1+z)^{3}+1-\Omega_{\mathrm{M}}\right]$. Thus this model is fully characterised by the parameters $H_{0}$ and $\Omega_{\mathrm{M}}$, which can be easily compared to the model independent parameters $H_{0}$ and $q_{0}$. Expression (3) is the basis of our model dependent fit and, in contrast to (1), holds for arbitrary redshift.

The effect of peculiar velocities on the redshift can be incorporated by realising that

$c\left(1+z_{\mathrm{obs}}\right)=c\left(1+z_{\mathrm{com}}\right)\left[1+\hat{\boldsymbol{e}}\left(\boldsymbol{v}_{\mathrm{pec}}-\boldsymbol{v}_{\odot}\right) / c+\ldots\right]$,

where $\hat{\boldsymbol{e}}$ denotes the direction of observation (unit vector) - light propagates along $-\hat{\boldsymbol{e}}$ - and the dots stand for higher order terms in the peculiar velocities. As shown by Bonvin et al. (2006a,b), it is expected that the peculiar motion of the Solar system w.r.t. the $\mathrm{CMB}$ is the dominant source of anisotropy in the Hubble diagram. Indeed the CMB dipole has its correspondence in SN Ia data (Riess et al. 1995). It is easily subtracted, assuming that the CMB dipole is entirely due to the Sun's motion w.r.t. the CMB. We take the WMAP 1yr value for the dipole: $(l, b)=$ $\left(263.85^{\circ} \pm 0.1^{\circ}, 48.25^{\circ} \pm 0.04^{\circ}\right)$ and $v_{\odot} / c=T_{1} / T_{0}=[(3.346 \pm$ $0.017) /(2.725 \pm 0.001)]=1.23 \times 10^{-3}$ (Bennett et al. 2003). At small redshifts it is sufficient to work with

$c z_{\mathrm{cmb}}=c z_{\mathrm{com}}+\hat{\boldsymbol{e}} \boldsymbol{v}_{\mathrm{pec}}+\ldots$

We assume that the peculiar velocities are uncorrelated with zero mean and variance $\sigma_{v}^{2}$.

For the purpose of our model independent analysis we define the deviation from the linear Hubble law

$\Delta \equiv \log \left(H_{0} d_{\mathrm{L}}\right)-\log \left(c z_{\mathrm{cmb}}\right)$,

where $\log =\log _{10}$. In the following $z_{\mathrm{cmb}}$ is denoted by $z$. Note that often the empty Universe model is plotted as a reference, but that model assumes already the Einstein equation and we would like to avoid in our first test any reference to the dynamics of the 
Table 1. Compilation of some characteristics of data sets and their subsamples used throughout this work.

\begin{tabular}{llccc}
\hline \hline Set & Reference(s) & $\begin{array}{c}\text { Full set } \\
\text { \#SNe }(\bar{z})\end{array}$ & $\begin{array}{c}A_{\mathrm{V}}<1 \\
\# \text { SNe }(\bar{z})\end{array}$ & $\begin{array}{c}A_{\mathrm{V}}<1, z<0.2 \\
\text { \#SNe }(\bar{z})\end{array}$ \\
\hline A & Tonry et al. 03 & $253(0.263)$ & $244(0.273)$ & $139(0.037)$ \\
& Barris et al. 04 & & & \\
B & Riess et al. 04 & $186(0.393)$ & $182(0.398)$ & $77(0.041)$ \\
C & Astier et al. 06 & $117(0.407)$ & $117(0.407)$ & $44(0.038)$ \\
D & Jha et al. 06 & $131(0.023)$ & $119(0.024)$ & $119(0.024)$ \\
\hline
\end{tabular}

Universe and stick to a purely kinematic test combined with the spatial information provided by the data.

The quantity $\Delta$ is free of the Hubble constant, once (1) or (3) are used. Equivalently, $\log \left(H_{0} d_{\mathrm{L}}\right)$ is related to the "Hubbleconstant-free" distance modulus

$m-\mathcal{M}=5 \log \left(H_{0} d_{\mathrm{L}}\right)$,

with $\mathcal{M} \equiv M-5 \log H_{0}+25$ (Perlmutter et al. 1999). However, to specify the $\mathrm{SN}$ data (in terms of $\log \left(H_{0} d_{\mathrm{L}}\right)$ or $\left.m-\mathcal{M}\right)$ a certain calibration has to be adopted, which makes use of fiducial values $H_{0}^{*}$ and $\mathcal{M}^{*}$.

In order to use $\mathrm{SNe}$ for the study of cosmology, one assumes that type Ia SNe can be made standard candles to a good approximation, i.e. $\mathcal{M}$ becomes a universal number. As this number depends mainly on the physics of SNe (the universality of $H_{0}$ is taken for being granted), it is treated as a nuissance parameter in the cosmological data analysis. Consequently, for the estimation of cosmological parameters one marginalises over $\mathcal{M}$ (or equivalently $H_{0}$ ). However, if we are interested in effects of large-scale structure formation or a test of the cosmological principle, we need to study the direction dependent off-set from the adopted calibration.

Below we follow the convention of Tonry et al. (2003), where a Hubble rate of $H_{0}^{*}=65 \mathrm{~km} \mathrm{~s}^{-1} \mathrm{Mpc}^{-1}$ is assumed; we thus define (the index $i$ denotes a particular $\mathrm{SN}$ )

$\Delta_{i} \equiv \log \left(H_{0}^{*} d_{\mathrm{L} i}\right)-\log \left(c z_{i}\right)$,

which is compared to the second order expression

$\Delta^{*}\left(z_{i}\right)=-\log \left(H_{0} / H_{0}^{*}\right)+\log \left[1+\left(1-q_{0}\right) \frac{z_{i}}{2}\right]$,

or the equivalent in case of the flat $\Lambda \mathrm{CDM}$ fits. We treat all data sets (see below) in the same way.

As we have no access to the absolute calibration of the $\mathrm{SNe}$, we cannot measure $H_{0}$. However, we can measure the ratio $H_{0} / H_{0}^{*}$, or $\mathcal{M}-\mathcal{M}^{*}=-5 \log \left(H_{0} / H_{0}^{*}\right)$, i.e. a calibration offset. Thus, a $10 \%$ effect in $H_{0} / H_{0}^{*}$ corresponds to 0.2 mag in $\mathcal{M}$. The difference $\mathcal{M}-\mathcal{M}^{*}$ seems to be better suited for the comparison of different data sets, whereas we work with $H_{0} / H_{0}^{*}$, which seems to us better suited for the study of cosmological anisotropies.

\section{Data}

We apply our tests on four different data sets that differ in sky and redshift coverage, shown in Figs. 1 and 2, as well as in systematics. A summary of the four data sets is given in Table 1 .

Our first data set (A) consists of $253 \mathrm{SNe}$ with redshifts $z \in[0.0023,1.755]$ (mean $\bar{z}=0.27$ ) from Tonry et al. (2003) and Barris et al. (2004). This data set has a fairly homogeneous
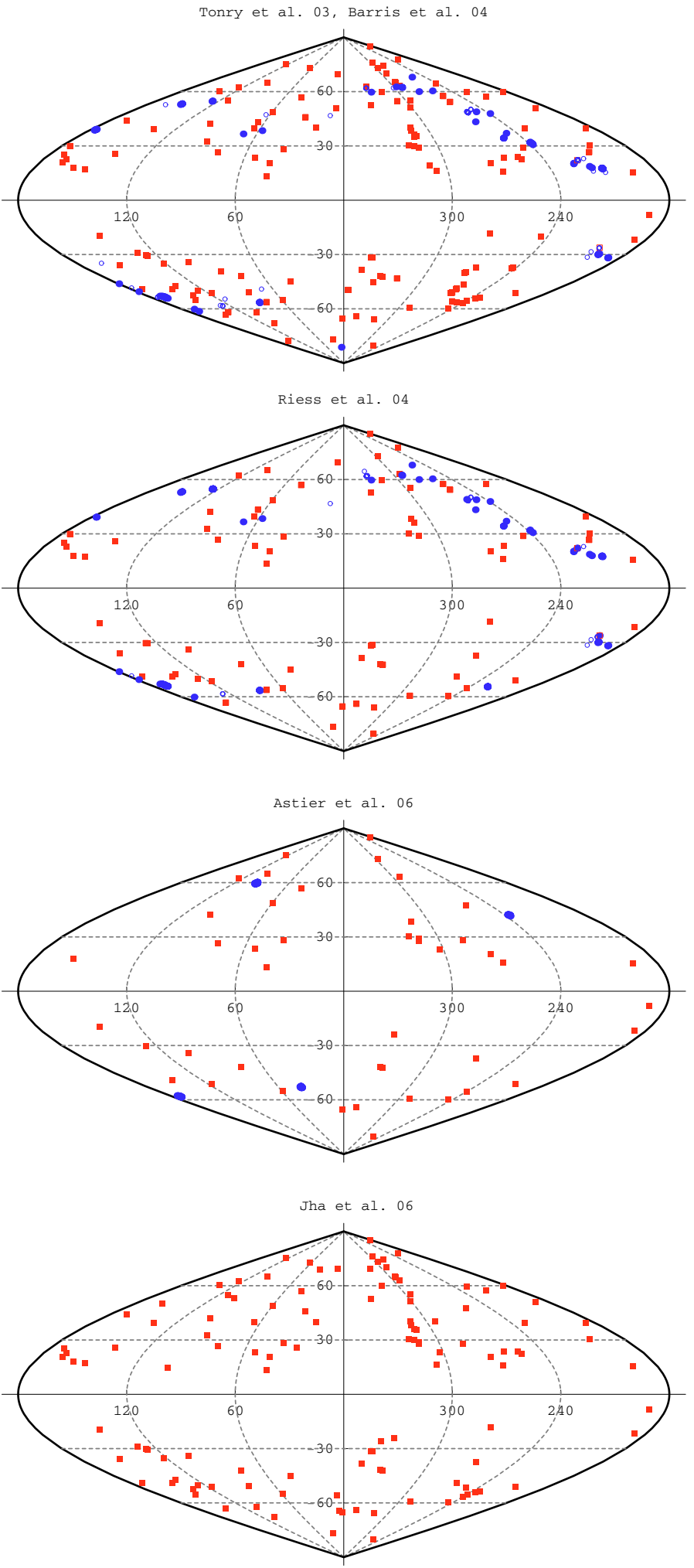

Fig. 1. Distribution of SNe Ia on the sky in galactic coordinates. Red squares denote $\mathrm{SNe}$ with $z<0.2$, whereas blue disks stand for $\mathrm{SNe}$ with $z>0.2$. Only $\mathrm{SNe}$ with extinction $A_{\mathrm{V}}<1$ are shown. For $\mathrm{SNe}$ indicated by open symbols no information on $A_{\mathrm{V}}$ is available. From top to bottom: 244 (105 with $z<0.2,139$ with $z>0.2)$ SNe Ia from Tonry et al. (2003) and Barris et al. (2004); 182 SNe Ia (77 with $z<0.2$ and 105 with $z>0.2$ ) from Riess et al. (2004); 117 SNe Ia (44 with $z<0.2$ and 73 with $z>0.2$ ) from Astier et al. (2006), one can easily spot the four SNLS fields of view (blue); 119 SNe Ia from Jha et al. (2006) all at small redshift. 

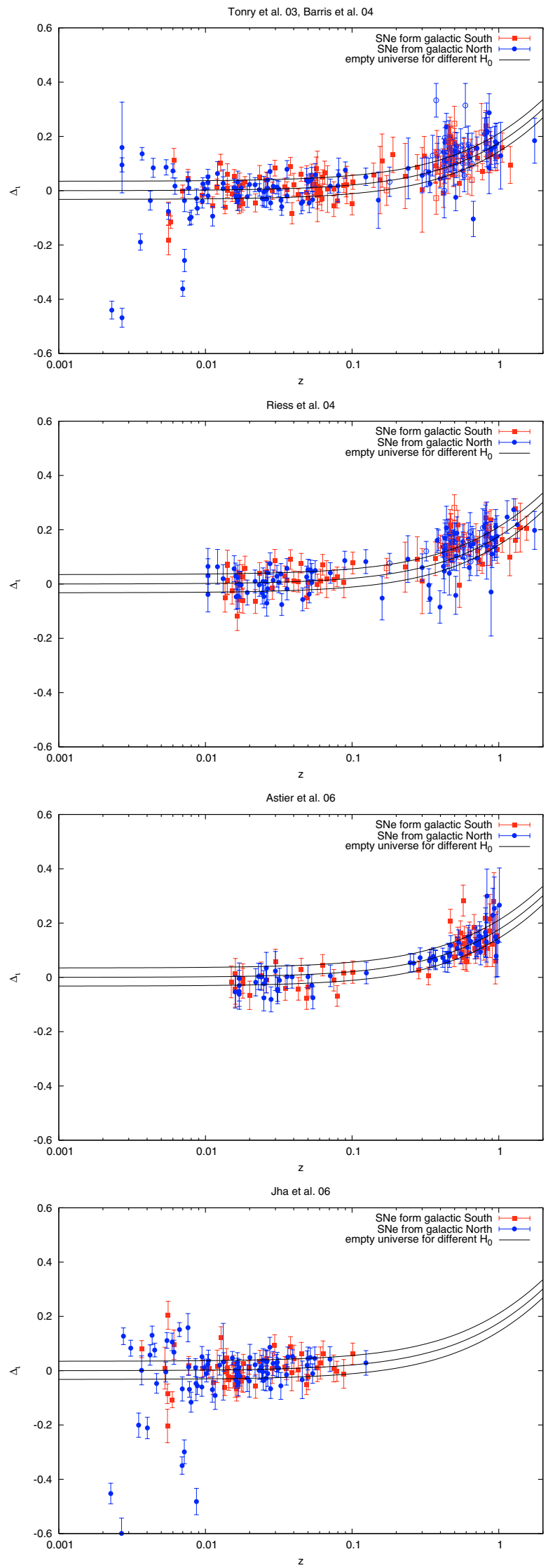

Fig. 2. Deviation from the linear Hubble law (see Eq. (7)) for the four data sets from Fig. 1. SNe from the North (South) galactic hemisphere are shown in blue (red). As a reference we show the expected deviation for the empty Universe model $(q=j=0 ; k=-1)$ with three different values of the SN calibration (black solid lines), corresponding to $H_{0} / H_{0}^{*}=0.85,1,1.15$ from top to bottom. sky coverage (except in the zone of avoidance) and is therefore suitable for our analysis. For each SN Ia we use the (galactic) coordinates, the distance modulus and the redshift in the CMB frame. We also use information on light extinction to exclude supernovae with $A_{\mathrm{V}}>1.0$ from our analysis. A subset of 42 $\mathrm{SNe}$ (originally from the Supernova Cosmology Project) has no information on $A_{\mathrm{V}}$ specified. We include the specified errors of the distance modulus and the redshift in our analysis and treat them as $1 \sigma$ errors. The errors on the distance modulus includes the error from photometry, the scatter between different analysis methods and an error from the intrinsic dispersion of SNe Ia.

The second data set (B) is from Riess et al. (2004), containing $186 \mathrm{SNe}$ with $z \in[0.0104,1.755]$ with mean $\bar{z}=0.40$. Data set $\mathrm{B}$ is not independent from data set $\mathrm{A}$, as it largely contains the same SNe. However, different corrections have been applied and different selection criteria have been used. A comparison of data set A and data set B can thus serve as an estimate of systematic errors in the SNe Ia data reduction. Data set B contains a "gold" and a "silver" set. We make use of the "silver" set here in order to have a good sky coverage. It should be stressed that the number of SNe with $z<0.2$ in set $\mathrm{B}$ is significantly smaller than in set A.

As a third data set $(\mathrm{C})$ we use the more recent SuperNova Legacy Survey (SNLS) release (Astier et al. 2006); $117 \mathrm{SNe}$, $z=[0.015,1.01]$ and $\bar{z}=0.41$. The $\mathrm{SNe}$ at high redshift are independent from sets $\mathrm{A}$ and $\mathrm{B}$, but come from four fields of view only (see Fig. 1). Thus we should expect that the pencil beam geometry of this data set is not ideal for the type of analysis that we have in mind, although it provides improved control over observational systematics and has very small errors compared to data set $\mathrm{A}$ and $\mathrm{B}$. At low redshifts, data set $\mathrm{C}$ relies largely on the same $\mathrm{SNe}$ as data set $\mathrm{A}$ and $\mathrm{B}$, but again with different processing and selection criteria, so it provides again a cross check for systematics. In contrast to the sets A and B, the intrinsic scatter of $\mathrm{SNe}$ is not included in the error provided by the SNLS team, rather it has to be incorporated as an additional term in the fits.

Finally, we also use the most recent compilation of "local" SNe from Jha et al. (2006) (data set D); $131 \mathrm{SNe}, z \in$ $[0.00226,0.1242]$ and $\bar{z}=0.024$. It largely contains the same $\mathrm{SNe}$ as data set $\mathrm{A}$, the main difference being a largely improved procedure to obtain the distance moduli and their errors. This set has a fairly good sky coverage, but is limited to small redshifts. Like for data set $\mathrm{C}$ the intrinsic scatter is not included in the provided error.

Two more recent data sets are not used because they contain SNe from very small regions of the sky only, i.e. from the two HST GOODS fields (Riess et al. 2006) and 20 fields of view within three hours in right ascension (ESSENCE collaboration: Wood-Vasey et al. 2007). These surveys suffer from the same limitations as the SNLS data, they are a set of pencil beams.

For all data sets we can see from Fig. 1 that the sky coverage is much better for the low redshift SN $(z<0.2)$. Figure 2 provides an impression of the redshift distribution of the samples for both galactic hemispheres. The current lack of SN observations at intermediate redshifts $(z=0.1$ to 0.3$)$ is easily spotted. At small $z<0.01$ the spreading of the distribution due to peculiar velocities of the $\mathrm{SNe}$ is seen. The deviation from the linear Hubble law becomes visible at $z>0.1$.

The four data sets used here differ from each other in the method applied to estimate the values of the fitted parameters. In order to fit the $\mathrm{SN}$ observations of set A, Tonry et al. (2003) and Barris et al. (2004) used four fit methods: the MLCS method described in Riess et al. (1998), the $\Delta m_{15}$ method (Philips et al. 1999) and its improved version dm15 
(Germany et al. 2001). Additionally, they used the BATM method by Tonry et al. (2003). For data set B, the BATM method was used to check the results with the improved version MLCS2k2 described in Jha (2002) and Jha et al. (2006). This method is also used by Jha et al. (2006) to fit the SNe of data set D. Astier et al. (2006) used the SALT method (Guy et al. 2005) to fit the data of set $\mathrm{C}$.

The reader should be aware that Tonry et al. (2003), Barris et al. (2004) and Riess et al. (2004) provide $z_{\mathrm{cmb}}$ for each SN, whereas Astier et al. (2006) and Jha et al. (2006) give $z_{\odot}$.

\section{Comparing hemispheres}

As motivated in the introduction, we wish to test the isotropy of the Hubble diagram. As the number of observed SNe is still small, it does not make sense to look into small-scale variations, rather we should look at the largest possible scales. The largest possible anisotropy scale is $180^{\circ}$. A very simple test is splitting the sky into hemispheres and to compare the corresponding Hubble diagrams. We can search for directions of anisotropies by rotating the respective poles over the sky. A similar study has been used by Eriksen et al. (2004) to analyse the cosmic microwave sky, which led to one of the several anomalies discovered in the WMAP data (see Copi et al. 2007, for a recent update and summary).

\subsection{Model-independent test of isotropy}

If we restrict the analysis to $\mathrm{SNe}$ at $z<0.2$, it is reasonable to fit the Hubble law (1) and its quadratic correction. This provides a model independent test of the cosmological principle and at the same time a check for systematic errors in the SN Ia search, observation and analysis.

In order to find the best-fitting values of the calibration $H_{0} / H_{0}^{*}$ and the deceleration $q_{0}$, we follow the standard approach and minimise

$\chi^{2}\left(H_{0} / H_{0}^{*}, q_{0}\right)=\sum_{i} \frac{\left[\Delta_{i}-\Delta^{*}\left(z_{i} ; H_{0} / H_{0}^{*}, q_{0}\right)\right]^{2}}{\sigma_{\Delta_{i}}^{2}+\sigma_{\text {int }}^{2}+\left(\sigma_{v}^{2}+\sigma_{z_{i}}^{2}\right) / z_{i}^{2}}$,

where the sum goes over a subset of one of the three data sets. For the data sets $\mathrm{A}$ and $\mathrm{B}$ the intrinsic dispersion $\sigma_{\text {int }}$ is included in $\sigma_{\Delta_{i}}$, for data set $\mathrm{C}$ and $\mathrm{D} \sigma_{\text {int }}>0 . \sigma_{v}$ and $\sigma_{z}$ denote the dispersion of the peculiar velocities and error of the redshift measurement. The latter two are most relevant for nearby SNe.

\subsubsection{Full sky}

As a reference we first determine the best fit values for $H_{0} / H_{0}^{*}$ and $q_{0}$ for the four SN Ia data sets. In doing so we aim at finding the best suited values for redshift and light extiction cuts, and we look a the best suited value of the peculiar velocity dispersion. We adopt a value of $\sigma_{v}=345 \mathrm{~km} \mathrm{~s}^{-1}$ for all four data sets, as it provides a reasonable $\chi^{2}$ per degree of freedom of order one for all data sets under consideration. The $z<0.2$ samples from sets A to D have mean redshifts of $0.038,0.041,0.038$ and 0.024 , respectively.

The results of the full-sky fits are presented in Fig. 3 (except for set $\mathrm{C}$ ) and in Table 2 . The fits shown in the figure correspond to the first fit in the table for the respective data set. We find that all four data sets provide fits that are consistent with each other. The largest difference is observed with respect to the SN calibration $\left(H_{0} / H_{0}^{*}\right)$ in data set $\mathrm{C}$. This happens because the SN

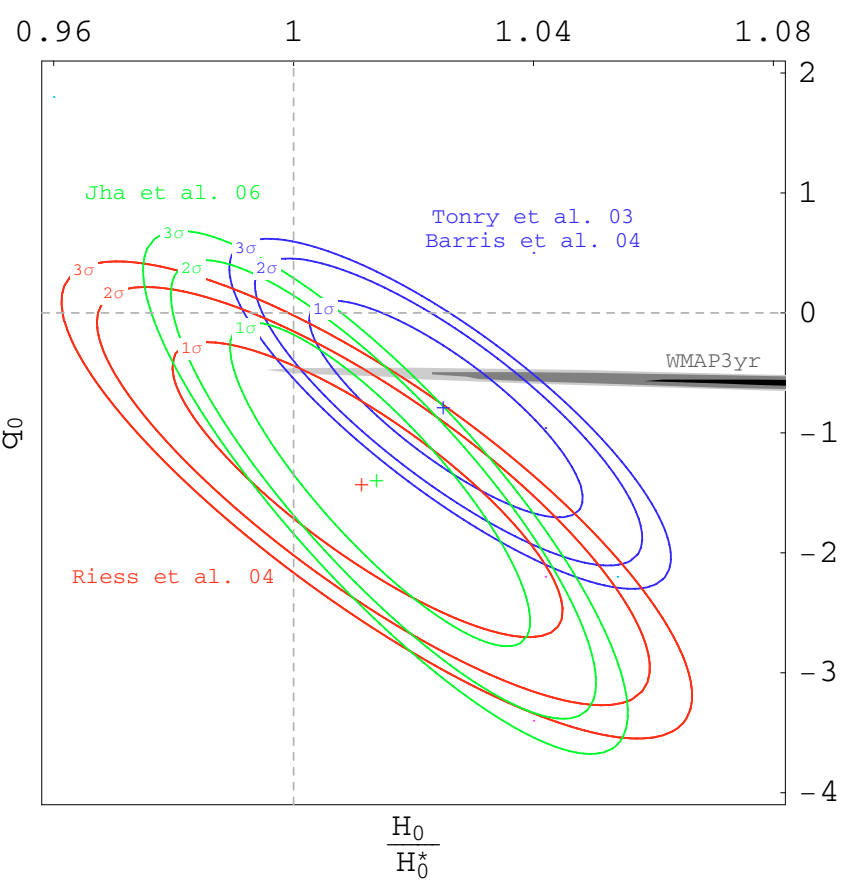

Fig. 3. Confidence contours for a model-independent full-sky fit to the Hubble law at second order for three SNe Ia data sets. SNe up to redshift $z=0.2$ are included in the fits. We determine the calibration off-set $H_{0} / H_{0}^{*}$ and the deceleration parameter $q_{0}$. As a comparison we show the corresponding contours of the WMAP measurement (Spergel et al. 2006). In contrast to the spread of the SN calibration off-set, its best-fit value has no physical relevance in the case of full sky fits. Thus the WMAP data may be moved horizontally in this figure.

calibration of that set has been fit to the WMAP measurement of $H_{0}$, whereas the other three data sets did not assume the WMAP value. At small redshifts, only data set $\mathrm{B}$ gives rise to a marginal evidence for acceleration. However, all data sets are consistent with acceleration and with the fit to WMAP data.

In order to test the robustness of the fits we vary the value of the parameters $A_{\mathrm{V}}, \sigma_{v}, \sigma_{\text {int }}$ and the range in redshift (see Table 2). The most significant effect comes from a restriction of the redshift range to $0.02<z<0.2$ or even $0.01<z<0.1$, which leads to a noticeable change in the best-fit value for $q_{0}$ and a substantial increase in its error. Shifting the maximal redshift to $z=0.3$ changes the result only slightly. From this point of view it is reasonable to include all SNe with $A_{\mathrm{V}}<1$ up to $z=0.2$ in order to fit the Hubble law up to the second order in redshift.

Although the full-sky fits indicate a robust data set C, our studies have shown that it is not well suited to be used within this work due to its small number of $\mathrm{SNe}$ and its bad sky coverage. We therefore focus on the results of data sets A, B and D in the following chapters.

\subsubsection{Galactic and equatorial hemispheres}

We start to study the anisotropy of the Hubble diagram by comparing those hemispheres which have a natural origin such as the galactic hemispheres and the equatorial hemispheres. The galaxy defines the zone of avoidance and is approximately North-South symmetric. Thus the galactic hemispheres cover approximately the same observable regions of the sky and should be well suited for a cosmological test. On the other hand, most observations (especially at small redshifts) are done from the ground and thus the equatorial system is distinguished. Any correlation with this 
Table 2. Robustness of the full-sky fit of the calibration $H_{0} / H_{0}^{*}$ and the deceleration parameter $q_{0}$ at small redshifts. We compare the number of degrees of freedom (d.o.f.), $\chi^{2} /$ d.o.f., and the best fit cosmological parameters for the four data sets described in the text for various assumptions on acceptable light extinction $A_{\mathrm{V}}$, peculiar velocity dispersion $\sigma_{v}$, intrinsic dispersion $\sigma_{\text {int }}$, as well as redshift interval included in the fit. Our analysis with $A_{\mathrm{V}}<1$ includes all SNe without information on $A_{\mathrm{V}}$, but we exclude those when investigating $A_{\mathrm{V}}<0.5$.

\begin{tabular}{|c|c|c|c|c|}
\hline & d.o.f. & $\frac{\chi^{2}}{\text { d.o.f. }}$ & $\overline{\frac{H_{0}}{H_{0}^{*}}}$ & $q_{0}$ \\
\hline \multicolumn{5}{|l|}{ data set $\mathbf{A}: 253 \mathrm{SNe}, z \in[0.002,1.755]$} \\
\hline$A_{\mathrm{V}} \leq 1, \sigma_{v}=345 \mathrm{~km} \mathrm{~s}^{-1}, z \leq 0.2$ & 137 & 1.27 & $1.02 \pm 0.02$ & $-0.78 \pm 0.90$ \\
\hline$A_{\mathrm{V}} \leq 0.5$ & 116 & 1.30 & $1.02 \pm 0.02$ & $-0.56 \pm 0.94$ \\
\hline$\sigma_{v}=230 \mathrm{~km} \mathrm{~s}^{-1}$ & 137 & 1.82 & $1.03 \pm 0.02$ & $-0.97 \pm 0.85$ \\
\hline$\sigma_{v}=460 \mathrm{~km} \mathrm{~s}^{-1}$ & 137 & 0.97 & $1.02 \pm 0.03$ & $-0.68 \pm 0.96$ \\
\hline$\sigma_{v}=690 \mathrm{~km} \mathrm{~s}^{-1}$ & 137 & 0.67 & $1.02 \pm 0.03$ & $-0.61 \pm 1.06$ \\
\hline $0.02<z \leq 0.2$ & 73 & 1.20 & $1.01 \pm 0.03$ & $-0.31 \pm 1.08$ \\
\hline$z \leq 0.1$ & 128 & 1.33 & $1.02 \pm 0.03$ & $-0.52 \pm 0.52$ \\
\hline $0.01<z \leq 0.1$ & 97 & 1.11 & $1.00 \pm 0.03$ & $0.28 \pm 1.25$ \\
\hline$z \leq 0.3$ & 142 & 1.23 & $1.02 \pm 0.02$ & $-0.65 \pm 0.73$ \\
\hline \multicolumn{5}{|l|}{ data set B: $186 \mathrm{SNe}, z \in[0.010,1.755]$} \\
\hline$A_{\mathrm{V}} \leq 1, \sigma_{v}=345 \mathrm{~km} \mathrm{~s}^{-1}, z \leq 0.2$ & 75 & 0.84 & $1.01 \pm 0.03$ & $-1.42 \pm 1.23$ \\
\hline$A_{\mathrm{V}} \leq 0.5$ & 66 & 0.79 & $1.00 \pm 0.04$ & $-1.36 \pm 1.79$ \\
\hline$\sigma_{v}=230 \mathrm{~km} \mathrm{~s}^{-1}$ & 75 & 0.92 & $1.01 \pm 0.03$ & $-1.43 \pm 1.20$ \\
\hline$\sigma_{v}=460 \mathrm{~km} \mathrm{~s}^{-1}$ & 75 & 0.75 & $1.01 \pm 0.03$ & $-1.40 \pm 1.25$ \\
\hline$\sigma_{v}=690 \mathrm{~km} \mathrm{~s}^{-1}$ & 75 & 0.60 & $1.01 \pm 0.04$ & $-1.35 \pm 1.33$ \\
\hline $0.02<z \leq 0.2$ & 50 & 0.95 & $1.01 \pm 0.04$ & $-1.27 \pm 1.36$ \\
\hline$z \leq 0.1$ & 70 & 0.84 & $1.02 \pm 0.05$ & $-2.16 \pm 2.21$ \\
\hline$z \leq 0.3$ & 80 & 0.81 & $1.00 \pm 0.03$ & $-0.78 \pm 0.89$ \\
\hline \multicolumn{5}{|l|}{ data set C: $117 \mathrm{SNe}, z \in[0.015,1.01]$} \\
\hline$\sigma_{v}=345 \mathrm{~km} \mathrm{~s}^{-1}, \sigma_{\mathrm{int}}=0.03, z \leq 0.2$ & 42 & 0.84 & $1.07 \pm 0.04$ & $-0.57 \pm 1.63$ \\
\hline$\sigma_{v}=230 \mathrm{~km} \mathrm{~s}^{-1}$ & 42 & 0.92 & $1.08 \pm 0.04$ & $-0.57 \pm 1.59$ \\
\hline$\sigma_{v}=460 \mathrm{~km} \mathrm{~s}^{-1}$ & 42 & 0.76 & $1.08 \pm 0.05$ & $-0.58 \pm 1.71$ \\
\hline$\sigma_{v}=690 \mathrm{~km} \mathrm{~s}^{-1}$ & 42 & 0.60 & $1.08 \pm 0.06$ & $-0.63 \pm 1.88$ \\
\hline $0.02<z \leq 0.2$ & 30 & 1.07 & $1.07 \pm 0.05$ & $-0.28 \pm 1.83$ \\
\hline$z \leq 0.1$ & 40 & 0.87 & $1.06 \pm 0.05$ & $0.16 \pm 2.15$ \\
\hline$z \leq 0.3$ & 46 & 0.80 & $1.08 \pm 0.03$ & $-0.56 \pm 0.58$ \\
\hline$\sigma_{\text {int }}=0$ & 42 & 3.73 & $1.09 \pm 0.03$ & $-0.82 \pm 0.75$ \\
\hline$\sigma_{\text {int }}=0.02$ & 42 & 1.40 & $1.08 \pm 0.04$ & $-0.62 \pm 1.27$ \\
\hline \multicolumn{5}{|l|}{ data set D: $131 \mathrm{SNe}, z \in[0.002,0.124]$} \\
\hline$A_{\mathrm{V}} \leq 1, \sigma_{v}=345 \mathrm{~km} \mathrm{~s}^{-1}, \sigma_{\text {int }}=0.016, z \leq 0.2$ & 117 & 1.37 & $1.01 \pm 0.03$ & $-1.39 \pm 1.35$ \\
\hline$A_{\mathrm{V}} \leq 0.5$ & 99 & 1.43 & $1.00 \pm 0.02$ & $-0.86 \pm 1.36$ \\
\hline$\sigma_{v}=230 \mathrm{~km} \mathrm{~s}^{-1}$ & 117 & 2.14 & $1.02 \pm 0.02$ & $-1.68 \pm 1.26$ \\
\hline$\sigma_{v}=460 \mathrm{~km} \mathrm{~s}^{-1}$ & 117 & 0.98 & $1.01 \pm 0.03$ & $-1.16 \pm 1.45$ \\
\hline$\sigma_{v}=690 \mathrm{~km} \mathrm{~s}^{-1}$ & 117 & 0.59 & $1.00 \pm 0.04$ & $-0.86 \pm 1.60$ \\
\hline $0.02<z \leq 0.2$ & 50 & 1.24 & $0.97 \pm 0.04$ & $0.11 \pm 1.59$ \\
\hline$z \leq 0.1$ & 115 & 1.39 & $1.02 \pm 0.03$ & $-1.49 \pm 1.71$ \\
\hline $0.01<z \leq 0.1$ & 84 & 1.08 & $1.00 \pm 0.03$ & $-0.81 \pm 1.78$ \\
\hline$\sigma_{\text {int }}=0$ & 117 & 1.64 & $1.01 \pm 0.02$ & $-1.30 \pm 1.20$ \\
\hline$\sigma_{\text {int }}=0.03$ & 117 & 1.05 & $1.02 \pm 0.03$ & $-1.51 \pm 1.66$ \\
\hline
\end{tabular}
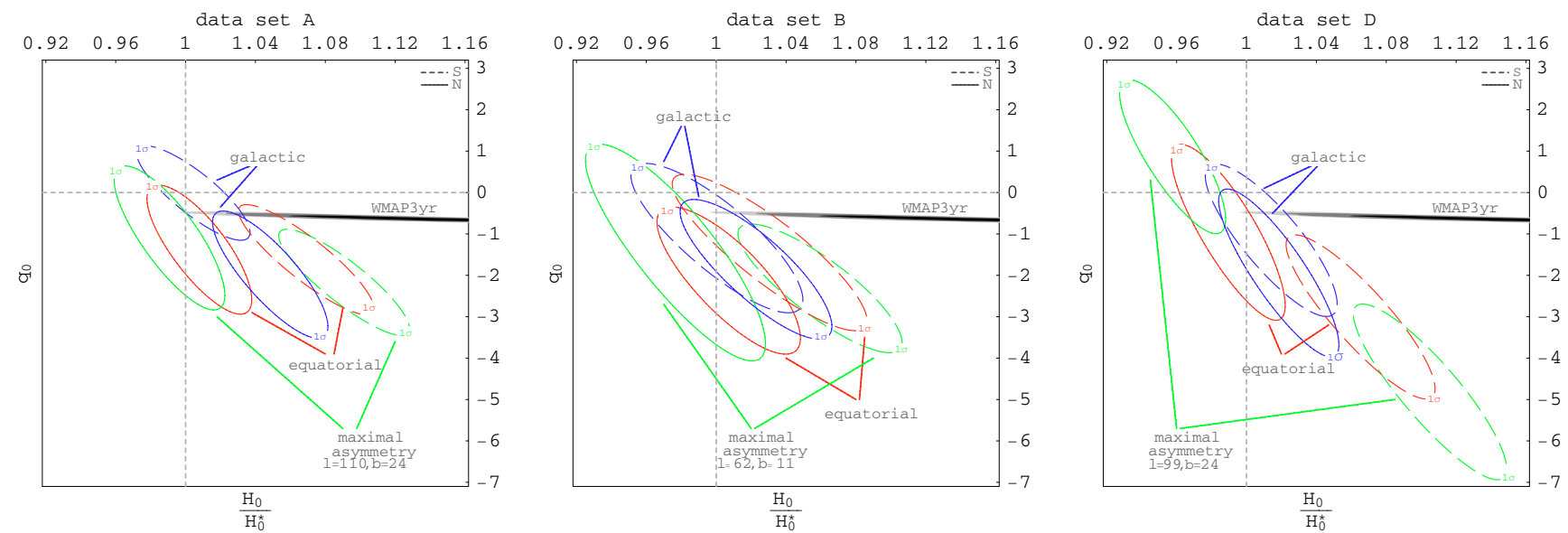

Fig. 4. North (full lines) and South (dashed lines) confidence contours and best-fit values for galactic, equatorial and maximum asymmetry hemispheres for the model-independent test. These fits should be compared to the full-sky fits of Fig. 3. We do not show results for data set C, as the number of SNe at $z<0.2$ of that data set turned out to be insufficient for this type of test. 
system would hint to a systematic effect in the search, observation or data analysis of SNe Ia.

Figure 4 shows the $1 \sigma$ contours for the galactic and equatorial hemispheres. As expected, all three data sets show a consistent fit for North and South galactic hemispheres. However, the equatorial system shows some unexpected deviations. For data set A we find a significant deviation of the calibration $H_{0} / H_{0}^{*}$ for the equatorial hemispheres. The northern hemisphere favours a lower value for $H_{0} / H_{0}^{*}$. The same trend is observed in data set $\mathrm{B}$, but from the analysis of data set $\mathrm{B}$ alone we would not pay attention. For data set $\mathrm{D}$ the deviation is again clearly seen and is not restricted to the calibration. The corresponding best fit values and their errors are displayed in Table 3.

In order to quantify the evidence for an equatorial NorthSouth asymmetry of the SN calibration, we use Monte Carlo (MC) simulations to check for artefacts of sky coverage. We test against 500 random realisations of the same data sets in which we mix the coordinates $l$ and $b$ for all SN Ia at $z<0.2$. For each simulated hemisphere pair we calculate the deviation in $\chi^{2}$ as

$\Delta \chi^{2} \equiv \min \left(\left|\chi_{N}^{2}(S)-\chi_{N}^{2}(N)\right|,\left|\chi_{S}^{2}(N)-\chi_{S}^{2}(S)\right|\right)$,

where $\chi_{N}^{2}(S)$ denotes the value of expression (9) for the best-fit parameters of the North hemisphere applied on the data of the South hemisphere, etc. We also calculate the deviation in $H_{0} / H_{0}^{*}$ and $q_{0}$ of the best-fit values from the opposite hemispheres. The results of these hemispherical fits are given in Table 3.

While no deviation from isotropy is found for data set B, we find data set A shows a statistically significant anisotropy in the equatorial system. Only $0.8 \%$ of our MCs give rise to a larger difference in $\Delta \chi^{2}$ and only $4.6 \%$ of the MCs show a larger difference in the calibration $H_{0} / H_{0}^{*}$. The latter number for data set $\mathrm{D}$ is $5.0 \%$. Thus, it seems the equatorial hemispheres do not agree with the expectation from the cosmological principle at the $95 \%$ C.L. with respect to the SN calibration (evidence from sets A and D).

\subsubsection{Hemispheres of maximal asymmetry}

We found evidence for a significant asymmetry in the equatorial coordinates in data sets $\mathrm{A}$ and $\mathrm{D}$ (and consistent with data set $\mathrm{B}$ ). We may ask if the asymmetry is maximised by the equatorial system or if more asymmetric directions exist on the sky. In order to test this anisotropy for its size we are going to identify those hemispheres which have the largest deviation in $\chi^{2}$.

We search for the maximal asymmetric hemispheres by rotating the poles across the sky. We calculate the deviation in $\chi^{2}$ for each hemisphere with poles at $l \in\left[0^{\circ}, 180^{\circ}\right]$ and $b \in$ $\left[-90^{\circ}, 90^{\circ}\right]$ in $1^{\circ}$ steps.

The results are given in Table 3 and the deviations in $\Delta \chi^{2}$ are illustrated in Fig. 5. Each coloured field in the figure is $10^{\circ} \times 10^{\circ}$ in size and its colour represents the mean deviation of the 100 corresponding pole positions. It is striking that all three data sets give rise to the same pattern on the sky (especially sets A and D), despite the fact that the methods of SN data reduction and selection criteria differ. By construction the pattern is symmetric and thus all information is contained in one hemisphere. In our discussion below we refer to the North galactic hemisphere.

The asymmetry is strongest in data set A, followed by D and B. As data set A contains more $\mathrm{SNe}$ than set $\mathrm{D}$, which contains more than set B, it seems that this trend is in accordance with the statistical power of the data sets. One can see, that two asymmetric directions are common in all three data sets. The first one is close to the equatorial poles at $(l, b)=\left(123^{\circ}, 27^{\circ}\right)$, the other one
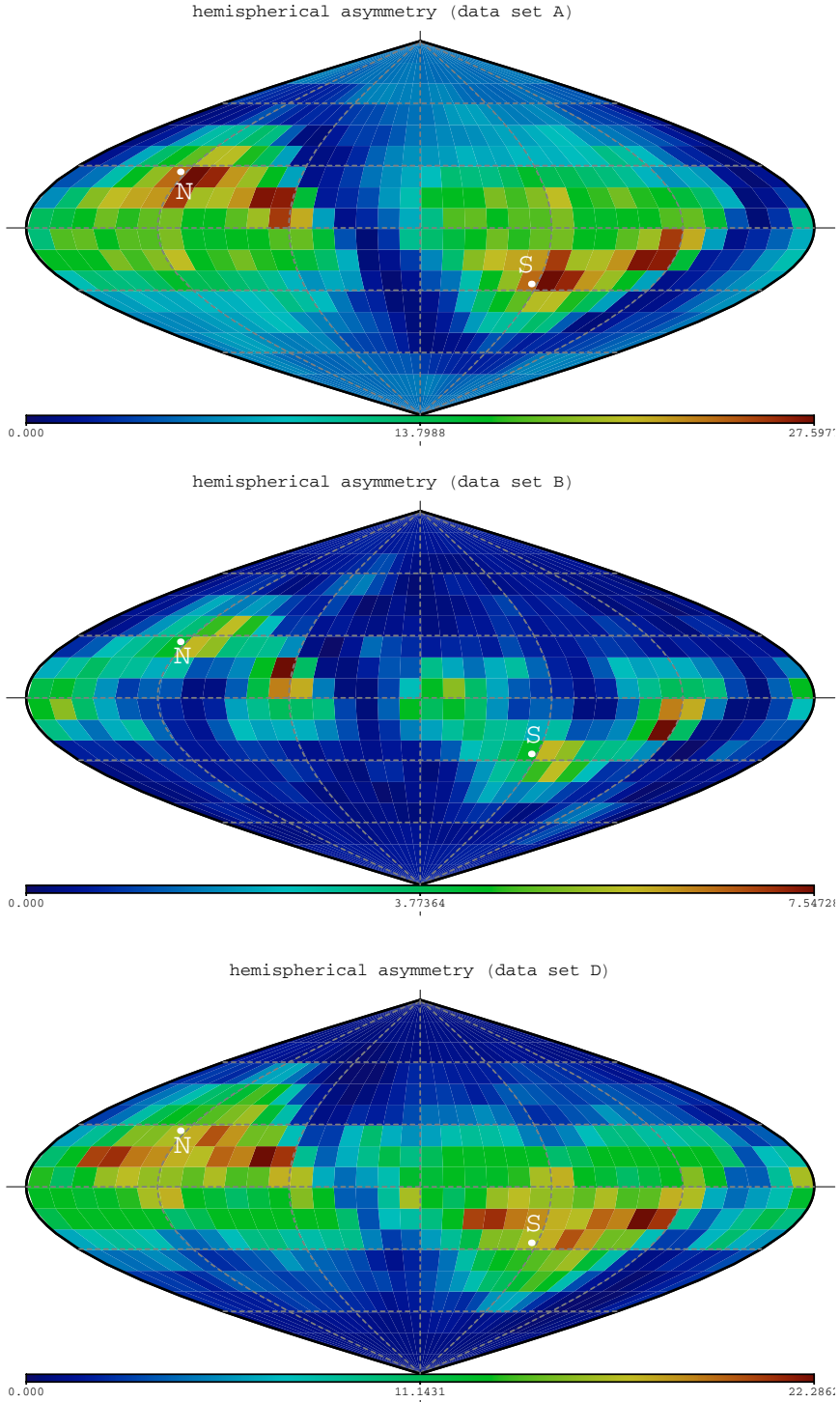

Fig. 5. Hemispherical asymmetry in $\Delta \chi^{2}$ for three SN Ia data sets at small redshift $(z<0.2)$ for the model independent fit. When the pole lies in a region with a bluish colour the asymmetry is small, the red spots denote the directions of large asymmetry. We plot directions in galactic coordinates. The white points indicate the zenit (equatorial North) and its antipode (equatorial South).

is close to $(l, b)=\left(70^{\circ}, 15^{\circ}\right)$. The proximity to the equatorial system is most pronounced in set A. Sets B and D maximise the asymmetry toward a direction $\sim\left(70^{\circ}, 5^{\circ}\right)$.

Let us also note that the observed pattern is unexpected. For randomly distributed $\mathrm{SNe}$ with a gaussian scatter in magnitude, we would expect that the resulting pattern would show less structure.

We performed $500 \mathrm{MC}$ simulations in order to check if the observed amount of asymmetry is to be expected for a data set with this kind of sky coverage. As above we mix the coordinates of the $\mathrm{SNe}$ of the data set under consideration and search for the maximal asymmetry in each of the simulated data sets. In order to keep the computational effort to a minimum, we now use a step size of $5^{\circ}$. The asymmetry of the MC data sets is compared to the one from the original set. The results are given in Table 4. The larger step size in the search for the maximal asymmetric 
Table 3. Hemisphere fit of $H_{0} / H_{0}^{*}$ and $q_{0}$ at small redshifts. We compare the number of degrees of freedom (d.o.f.), $\chi^{2} /$ d.o.f., and the best fit cosmological parameters for the data sets A, B and D for the galactic, equatorial and maximal asymmetry hemispheres. In addition we show for the galactic and equatorial hemispheres the percentage of $\mathrm{MC}$ simulations with a larger deviation in $\chi^{2}, H_{0} / H_{0}^{*}$ and $q_{0}$. In brackets we provide the respective differences $\Delta \chi^{2}, \Delta \frac{H_{0}}{H_{0}^{*}}$ and $\Delta q_{0}$.

\begin{tabular}{|c|c|c|c|c|c|c|c|}
\hline & d.o.f. & $\frac{\chi^{2}}{\text { d.o.f. }}$ & $\frac{H_{0}}{H_{0}^{*}}$ & $q_{0}$ & $\begin{array}{l}\mathrm{MC}_{\chi^{2}} \\
\left(\Delta \chi^{2}\right)\end{array}$ & $\begin{array}{c}\mathrm{MC}_{\frac{H_{0}}{H_{0}^{*}}} \\
\left(\Delta \frac{H_{0}}{H_{0}^{*}}\right)\end{array}$ & $\begin{array}{l}\mathrm{MC}_{q_{0}} \\
\left(\Delta q_{0}\right)\end{array}$ \\
\hline \multicolumn{8}{|l|}{ data set $\mathrm{A}$} \\
\hline \multicolumn{7}{|l|}{ Galactic hemispheres } & \\
\hline North: $(l, b)=\left(0^{\circ}, 90^{\circ}\right)$ & 72 & 1.34 & $1.05 \pm 0.03$ & $-1.92 \pm 1.53$ & $32.6 \%$ & $21.2 \%$ & $14.2 \%$ \\
\hline South: $(l, b)=\left(0^{\circ},-90^{\circ}\right)$ & 63 & 1.18 & $1.00 \pm 0.03$ & $0.03 \pm 1.14$ & $(4.75)$ & $(0.05)$ & $(1.95)$ \\
\hline \multicolumn{8}{|l|}{ Equatorial hemispheres } \\
\hline North: $(l, b)=\left(123^{\circ}, 27^{\circ}\right)$ & 70 & 1.35 & $1.01 \pm 0.03$ & $-1.31 \pm 1.63$ & $0.8 \%$ & $4.6 \%$ & $81.6 \%$ \\
\hline South: $(l, b)=\left(303^{\circ},-27^{\circ}\right)$ & 65 & 1.07 & $1.07 \pm 0.04$ & $-1.57 \pm 1.38$ & $(21.71)$ & $(0.06)$ & $(0.26)$ \\
\hline \multicolumn{8}{|l|}{ Hemispheres max. Asymmetry in $\chi^{2}$ : } \\
\hline Pole: $(l, b)=\left(110^{\circ}, 24^{\circ}\right)$ & 65 & 0.89 & $0.99 \pm 0.03$ & $-1.02 \pm 1.75$ & & & \\
\hline Pole: $(l, b)=\left(290^{\circ},-24^{\circ}\right)$ & 70 & 1.38 & $1.09 \pm 0.04$ & $-2.12 \pm 1.29$ & & & \\
\hline \multicolumn{8}{|l|}{ data set $B$} \\
\hline $\begin{array}{l}A_{\mathrm{V}} \leq 1, \sigma_{v}=345 \mathrm{~km} \mathrm{~s}^{-1}, z \leq 0.2 \\
\text { Galactic hemispheres }\end{array}$ & 75 & 0.84 & $1.01 \pm 0.03$ & $-1.42 \pm 1.23$ & & & \\
\hline North: $(l, b)=\left(0^{\circ}, 90^{\circ}\right)$ & 41 & 0.80 & $1.02 \pm 0.04$ & $-1.75 \pm 1.69$ & $81.4 \%$ & $56.2 \%$ & $59.8 \%$ \\
\hline South: $(l, b)=\left(0^{\circ},-90^{\circ}\right)$ & 32 & 0.93 & $1.00 \pm 0.05$ & $-0.99 \pm 1.80$ & $(0.49)$ & $(0.02)$ & $(0.76)$ \\
\hline \multicolumn{8}{|l|}{ Equatorial hemispheres } \\
\hline North: $(l, b)=\left(123^{\circ}, 27^{\circ}\right)$ & 41 & 0.80 & $1.01 \pm 0.04$ & $-2.02 \pm 1.79$ & $15.2 \%$ & $54.2 \%$ & $61.6 \%$ \\
\hline South: $(l, b)=\left(303^{\circ},-27^{\circ}\right)$ & 32 & 0.86 & $1.03 \pm 0.06$ & $-1.32 \pm 1.85$ & $(5.36)$ & $(0.02)$ & $(0.70)$ \\
\hline \multicolumn{8}{|l|}{ Hemispheres max. Asymmetry in $\chi^{2}$ : } \\
\hline Pole: $(l, b)=\left(62^{\circ}, 11^{\circ}\right)$ & 33 & 0.57 & $0.97 \pm 0.05$ & $-1.26 \pm 2.62$ & & & \\
\hline Pole: $(l, b)=\left(242^{\circ},-11^{\circ}\right)$ & 40 & 0.93 & $1.08 \pm 0.05$ & $-2.23 \pm 1.56$ & & & \\
\hline \multicolumn{8}{|l|}{ data set $D$} \\
\hline $\begin{array}{l}A_{\mathrm{V}} \leq 1, \sigma_{v}=345 \mathrm{~km} \mathrm{~s}^{-1}, \sigma_{\text {int }}=0.016 z \leq 0.2 \\
\text { Galactic hemispheres }\end{array}$ & \multicolumn{6}{|c|}{ Galactic hemispheres } & \\
\hline North: $(l, b)=\left(0^{\circ}, 90^{\circ}\right)$ & 69 & 1.49 & $1.02 \pm 0.03$ & $-1.85 \pm 2.03$ & $82.8 \%$ & $89.4 \%$ & $73.6 \%$ \\
\hline South: $(l, b)=\left(0^{\circ},-90^{\circ}\right)$ & 46 & 1.25 & $1.01 \pm 0.04$ & $-1.07 \pm 1.84$ & $(0.82)$ & $(0.004)$ & $(0.77)$ \\
\hline \multicolumn{8}{|l|}{ Equatorial hemispheres } \\
\hline North: $(l, b)=\left(123^{\circ}, 27^{\circ}\right)$ & 64 & 1.71 & $0.99 \pm 0.03$ & $-0.85 \pm 2.12$ & $7.8 \%$ & $5.0 \%$ & $31.8 \%$ \\
\hline South: $(l, b)=\left(303^{\circ},-27^{\circ}\right)$ & 51 & 0.87 & $1.06 \pm 0.04$ & $-2.92 \pm 1.98$ & $(10.84)$ & $(0.075)$ & $(2.07)$ \\
\hline \multicolumn{8}{|l|}{ Hemispheres max. Asymmetry in $\chi^{2}$ : } \\
\hline Pole: $(l, b)=\left(99^{\circ}, 24^{\circ}\right)$ & 61 & 1.15 & $0.96 \pm 0.03$ & $0.96 \pm 1.85$ & & & \\
\hline Pole: $(l, b)=\left(279^{\circ},-24^{\circ}\right)$ & 54 & 1.31 & $1.10 \pm 0.04$ & $-4.73 \pm 2.14$ & & & \\
\hline
\end{tabular}

direction explains why the directions of maximal asymmetry differ from the ones in Table 3. For data sets A and D the asymmetry is larger than in $99.8 \%$ of our MCs. The deviation in the SN calibration seems to be significant at $>96 \%$ C.L. Data set D shows also a significant asymmetry in the extracted values of the deceleration parameter. Only $1.8 \%$ of the MCs show a larger difference. In contrast, the asymmetry in data set B appears not to be statistically significant. However, we should keep in mind that this data set shows a very similar asymmetry pattern but contains less $\mathrm{SNe}$ than sets A and D.

\subsubsection{Summary of model-independent test}

For data set A we have identified a statistically significant asymmetry in the equatorial system. It turns out to be due to an off-set in the calibration $H_{0} / H_{0}^{*}$ among the two hemispheres. The direction of maximal asymmetry is very close to that direction in data set A. Data set B does not show the same amount of asymmetry, but is qualitatively consistent with data set A. However, it contains less $\mathrm{SNe}$ and it is thus expected that the asymmetry should be less obvious. The larger data set D shows again a statistically significant asymmetry in the SN calibration.

We cannot offer an explanation for the asymmetry, but we think that the proximity of the direction of maximal asymmetry to the equatorial poles suggests an systematic error in one of the steps (search, observation, data analysis) to the calibration of $\mathrm{SNe}$.

A second direction of asymmetry has been identified in all three data sets. Its detection is statistically less significant.

\subsection{Isotropy in the flat $\Lambda C D M$ model}

Let us now include SNe Ia at any redshift in our test. This allows us to the test the concordance model of cosmology. We restrict our analysis to the flat $\Lambda \mathrm{CDM}$ model. Thus, the Hubble diagram can now be used to fit $\Omega_{\mathrm{M}}$ and $H_{0} / H_{0}^{*}$, the calibration off-set of SNe Ia. Here we do not make use of data set $\mathrm{D}$, as it is limited to small redshifts. 
Table 4. Hemispheres of maximal asymmetry from a $5^{\circ}$ search (in contrast to the more accurate $1^{\circ}$ search in Table 3 ). The purpose of the sparse search is to run MC simulations. The meaning of the columns is explained in the caption of Table 3 .

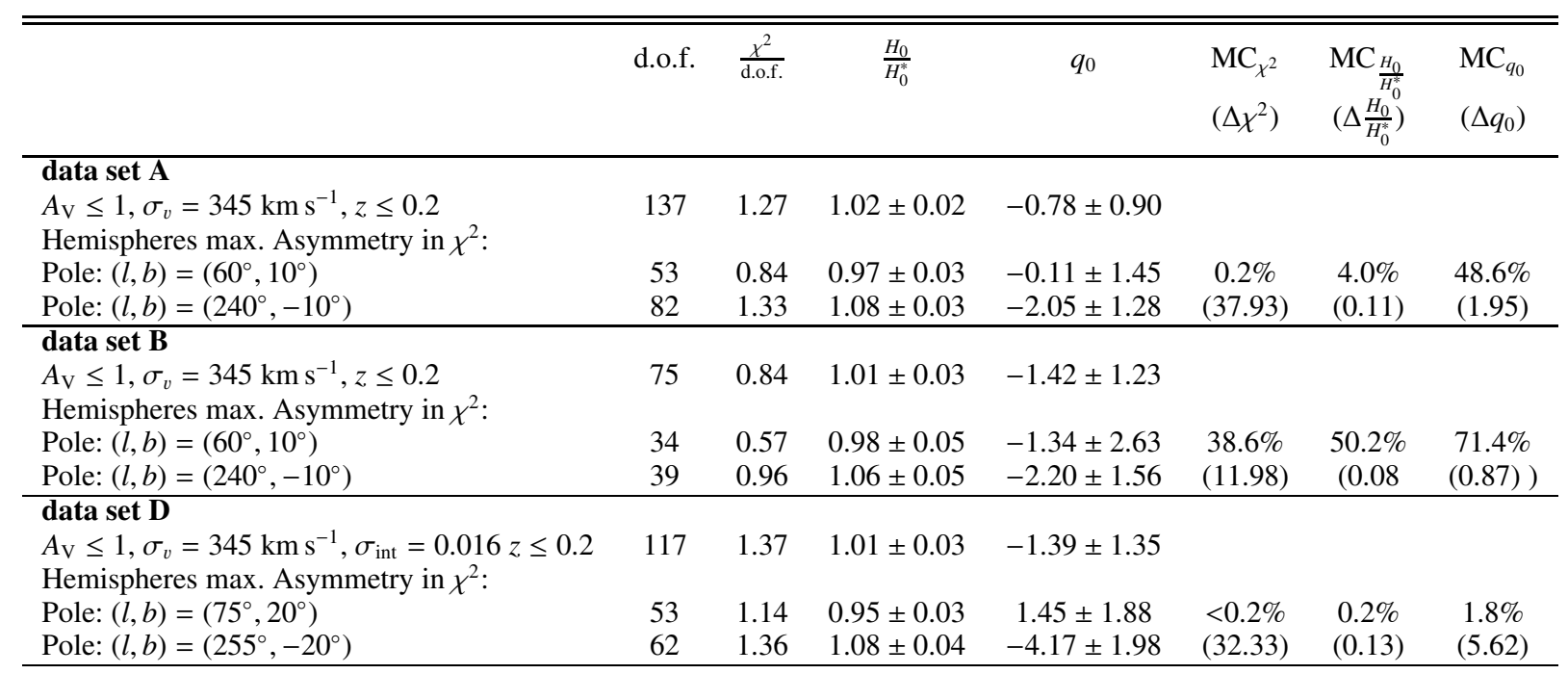

Table 5. Robustness of the full-sky fit to the flat $\Lambda \mathrm{CDM}$ model at arbitrary redshift. The fit parameters are the calibration $H_{0} / H_{0}^{*}$ and the dimensionless matter density $\Omega_{\mathrm{M}}$. We compare the number of degrees of freedom (d.o.f.), $\chi^{2} /$ d.o.f., and the best fit cosmological parameters for data sets $\mathrm{A}, \mathrm{B}$, and $\mathrm{C}$ for various assumptions on the acceptable light extinction $A_{\mathrm{V}}$, peculiar velocity dispersion $\sigma_{v}$, intrinsic dispersion $\sigma_{\text {int }}$ and redshift interval included in the fit.

\begin{tabular}{lrrrr}
\hline \hline & d.o.f. & $\frac{\chi^{2}}{\text { d.o.f. }}$ & $\frac{H_{0}}{H_{0}^{*}}$ & $\Omega_{\mathrm{M}}$ \\
\hline data set A: $253 \mathrm{SNe}, z \in[0.002,1.755]$ & 242 & 1.31 & $1.02 \pm 0.02$ & $0.31 \pm 0.06$ \\
$A_{\mathrm{V}} \leq 1, \sigma_{v}=345 \mathrm{~km} \mathrm{~s}^{-1}, z$ arbitrary & 242 & 1.62 & $1.02 \pm 0.02$ & $0.31 \pm 0.06$ \\
$\sigma_{v}=230 \mathrm{~km} \mathrm{~s}^{-1}$ & 242 & 1.14 & $1.01 \pm 0.02$ & $0.34 \pm 0.06$ \\
$\sigma_{v}=460 \mathrm{~km} \mathrm{~s}^{-1}$ & 242 & 0.96 & $1.01 \pm 0.02$ & $0.34 \pm 0.07$ \\
$\sigma_{v}=690 \mathrm{~km} \mathrm{~s}^{-1}$ & 137 & 1.27 & $1.03 \pm 0.03$ & $0.04_{-0.04}^{+0.66}$ \\
$0.0<z \leq 0.2$ & 211 & 1.20 & $1.01 \pm 0.02$ & $0.34 \pm 0.07$ \\
$0.01<z$ & 219 & 1.26 & $1.01 \pm 0.02$ & $0.32 \pm 0.07$ \\
$A_{\mathrm{V}} \leq 0.5$ & & & & \\
\hline data set B: $186 \mathrm{SNe}, z \in[0.010,1.755]$ & 180 & 1.11 & $0.98 \pm 0.02$ & $0.32 \pm 0.06$ \\
$A_{\mathrm{V}} \leq 1, \sigma_{v}=345 \mathrm{~km} \mathrm{~s}^{-1}, z$ arbitrary & 180 & 1.14 & $0.98 \pm 0.02$ & $0.32 \pm 0.06$ \\
$\sigma_{v}=230 \mathrm{~km} \mathrm{~s}^{-1}$ & 180 & 1.07 & $0.98 \pm 0.02$ & $0.32 \pm 0.06$ \\
$\sigma_{v}=460 \mathrm{~km} \mathrm{~s}^{-1}$ & 180 & 1.00 & $0.98 \pm 0.02$ & $0.32 \pm 0.06$ \\
$\sigma_{v}=690 \mathrm{~km} \mathrm{~s}^{-1}$ & 75 & 0.84 & $1.00 \pm 0.02$ & $\leq 0.96(2 \sigma)$ \\
$0.0<z \leq 0.2$ & 159 & 0.94 & $0.97 \pm 0.02$ & $0.32 \pm 0.06$ \\
$A_{\mathrm{V}} \leq 0.5$ & & & & \\
\hline data set C: $117 \mathrm{SNe}, z \in[0.015,1.01]$ & 115 & 1.02 & $1.08 \pm 0.03$ & $0.25 \pm 0.07$ \\
$A_{\mathrm{V}} \leq 1, \sigma_{v}=345 \mathrm{~km} \mathrm{~s}^{-1}, \sigma_{\text {int }}=0.03, z$ arbitrary & 115 & 1.05 & $1.08 \pm 0.05$ & $0.23 \pm 0.07$ \\
$\sigma_{v}=230 \mathrm{~km} \mathrm{~s}^{-1}$ & 115 & 0.99 & $1.08 \pm 0.02$ & $0.23 \pm 0.06$ \\
$\sigma_{v}=460 \mathrm{~km} \mathrm{~s}^{-1}$ & 115 & 0.93 & $1.08 \pm 0.03$ & $0.23 \pm 0.06$ \\
$\sigma_{v}=690 \mathrm{~km} \mathrm{~s}^{-1}$ & 42 & 0.84 & $1.08 \pm 0.04$ & $0.20_{-0.20}^{+1.48}$ \\
$0.0<z \leq 0.2$ & 115 & 8.60 & $1.08 \pm 0.01$ & $0.27 \pm 0.03$ \\
$\sigma_{\text {int }}=0$ & 115 & 1.68 & $1.08 \pm 0.05$ & $0.24 \pm 0.02$ \\
$\sigma_{\text {int }}=0.02$ & & & &
\end{tabular}

\subsubsection{Full sky}

To start with, we show the results for the full-sky fit in Table 5. Again we see that the fits are quite robust, except for the restriction to $z<0.2$. In that case, a matterless Universe $\left(\Omega_{\mathrm{M}}=0\right)$ cannot be ruled out. The confidence contours for the $\Lambda$ CDM fits to $\mathrm{SNe}$ Ia at arbitrary redshift are shown in Fig. 6. Only for data set $\mathrm{C}$ the best fit value is close to the one of the WMAP measurement. This is due to the fact that the $\mathrm{SN}$ calibration in set $\mathrm{C}$ was chosen to agree with WMAP measurements, whereas for $\mathrm{SNe}$ sets $\mathrm{A}$ and $\mathrm{B}$ no information from the $\mathrm{CMB}$ was used. In the following we restrict our presentation to data sets $\mathrm{A}$ and $\mathrm{B}$, as the pencil beam geometry of data set $\mathrm{C}$ turned out not to be suitable for our tests.

\subsubsection{Galactic and equatorial hemispheres}

The fits to North and South galactic and equatorial hemispheres are presented in Table 6 . The asymmetry in equatorial 


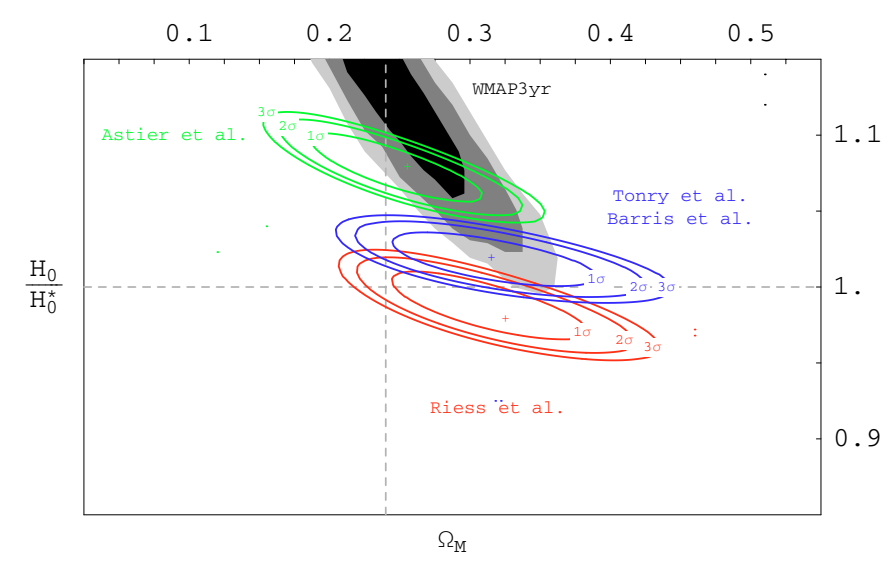

Fig. 6. Confidence contours for a full-sky fit to the flat $\Lambda \mathrm{CDM}$ model for three $\mathrm{SNe}$ Ia data sets. All SNe with $A_{\mathrm{V}}<1$ are included in the fits. We determine the calibration off-set $H_{0} / H_{0}^{*}$ and the dimensionless mass density $\Omega_{\mathrm{M}}$. For comparison we show the corresponding contours of the WMAP measurement (Spergel et al. 2006). For the latter $H_{0}$ indeed is the extracted Hubble parameter. Here the best-fit value of the calibration off-set is of no relevance, the vertical extension of the contours however reflects the physical scatter of the data.

coordinates found at low $z$ is confirmed by the full data sets A and B. Again we run $500 \mathrm{MCs}$ to test the statistical significance and find that the calibration off-set in the equatorial coordinates is now significant at $>95 \%$ C.L. in both data sets. In data set A it is at $>99 \%$ C.L. The corresponding confidence contours are shown in Fig. 7.

Additionally, we find now that data set A shows also asymmetry in galactic coordinates, which is an asymmetry in the extracted matter density at 95\% C.L.; see Table 6 for the strongly deviant values of $\Omega_{\mathrm{M}}$ in the North and South hemispheres, $\left|\Delta \Omega_{\mathrm{M}}\right|=0.2$. This asymmetry is not at an significant level in data set $\mathrm{B}$, but as for the model-independent test, data set B is fully consistent with the conclusions from data set A.

\subsubsection{Hemispheres of maximal asymmetry}

Finally, we search again for the maximally asymmetric pair of hemispheres. In Fig. 8 we show the hemispherical asymmetry $\Delta \chi^{2}$ in galactic coordinates. For data set $\mathrm{A}$, the location of the maxima is very similar to the location of maxima in Fig. 5, and we confirm our findings above.

In contrast, data set B shows a new structure, with a significant maximum close to $(l, b) \sim\left(235^{\circ}, 15^{\circ}\right)$. This direction does not reflect any obvious large scale structure, but as we average over half of the sky, the pointing is not expected to be precise. However, an inspection of the asymmetry in the number of degrees of freedom (d.o.f.) of the hemispheres shows that for data set $\mathrm{B}$ the maximum asymmetry in $\chi^{2}$ coincides with the maximal asymmetry in the number of d.o.f.. We conclude that the pattern for data set B is due to the geometry of the SN sample and is fully consistent with an isotropic Hubble diagram. However, this finding does not rule out the precence of the effect observed in data set A for two reasons: data set A contains significantly more $\mathrm{SNe}$ and is dominated by nerby $\mathrm{SNe}(\bar{z}=0.27)$, whereas set $\mathrm{B}$ is dominated by objects at higher redshifts $(\bar{z}=0.40)$.

As above we run MCs to quantify the statistical significance of our findings. It turns out that the pattern and amount of asymmetry in set B is not unexpected, still data set $\mathrm{A}$ is unexpected at the $95 \%$ C.L. (see Table 6).

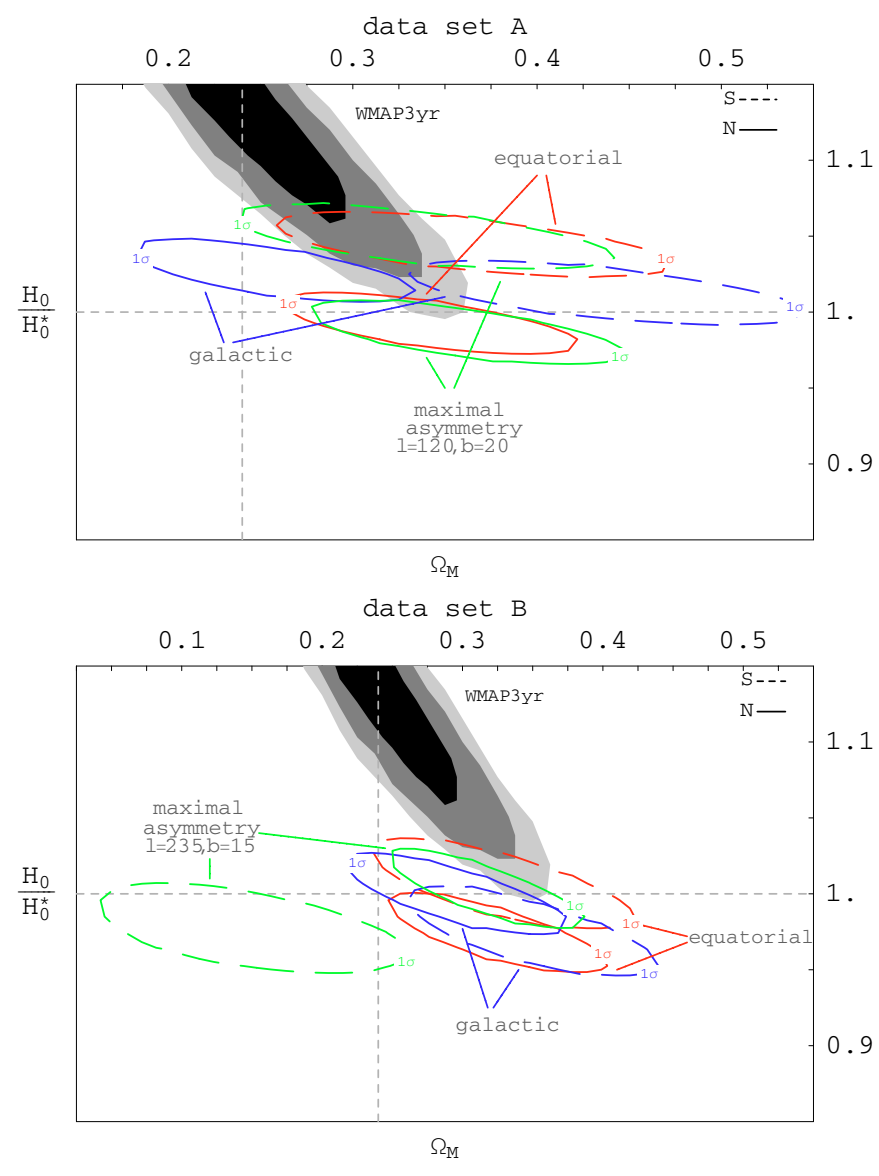

Fig. 7. North (full lines) and South (dashed lines) confidence contours and best-fit values for galactic, equatorial and maximum asymmetry hemispheres for the $\Lambda \mathrm{CDM}$ fit. These fits should be compared to the full-sky fits of Fig. 6. We do not show results for data set C, as the pencil beam geometry of that data set is not suitable for our test.

\subsubsection{Summary of the $\Lambda$ CDM test}

We confirm the findings of the model-independent test, namely a significant asymmetry in the $\mathrm{SN}$ calibration $\left(H_{0} / H_{0}^{*}\right)$ between North and South equatorial hemispheres. On top of that asymmetries show up (especially in set B) that seem to be linked to the geometry of the samples. We do not find significant evidence for large scale structure effects at the largest scales $\left(180^{\circ}\right)$, but as objects like the Shapely supercluster are significantly smaller, our test is not well suited to identify such structures.

\section{Results}

\subsection{Is the Hubble diagram isotropic?}

We argue above that we find statistically significant deviations of Hubble diagrams form isotropy. These are most significant in data sets $\mathrm{A}$ and $\mathrm{D}$, which contain most SNe. These asymmetries cannot be explained by the peculiar motion of the observer, as our analysis is done in the CMB rest-frame. If the asymmetry would be caused by peculiar motions of the SNe hosts, we would expect that its significance decreases when we include $\mathrm{SNe}$ at higher redshifts. This is not the case. As we go from the model-independent test at small redshifts $(z<0.2)$ to the model dependent test for arbitrary redshift, the significance of the effect is actually increased. On top of that, the magnitude of 
Table 6. Comparison of galactic, equatorial and maximal asymmetry hemispheres for the flat $\Lambda \mathrm{CDM}$ model. We use all $\mathrm{SNe}$ with $A_{\mathrm{V}}<1.0$ from data sets $\mathrm{A}$ and $\mathrm{B}$. The meaning of the columns is analogue to Table 3 .

\begin{tabular}{|c|c|c|c|c|c|c|c|}
\hline & d.o.f. & $\frac{\chi^{2}}{\text { d.o.f. }}$ & $\frac{H_{0}}{H_{0}^{*}}$ & $\Omega_{\mathrm{M}}$ & $\begin{array}{l}\mathrm{MC}_{\chi^{2}} \\
\left(\Delta \chi^{2}\right)\end{array}$ & $\begin{array}{l}\mathrm{MC}_{\frac{H_{0}}{H_{0}^{*}}} \\
\left(\Delta \frac{H_{0}}{H_{0}^{*}}\right)\end{array}$ & $\begin{array}{l}\mathrm{MC}_{\Omega_{\mathrm{M}}} \\
\left(\Delta \Omega_{\mathrm{M}}\right)\end{array}$ \\
\hline $\begin{array}{l}\text { data set } \mathbf{A} \\
A_{\mathrm{V}} \leq 1, \sigma_{v}=345 \mathrm{~km} \mathrm{~s}^{-1}, z \text { arbitrary } \\
\text { Galactic hemispheres }\end{array}$ & 242 & 1.31 & $1.02 \pm 0.02$ & $0.31 \pm 0.06$ & & & \\
\hline North: $(l, b)=\left(0^{\circ}, 90^{\circ}\right)$ & 136 & 1.36 & $1.02 \pm 0.02$ & $0.24 \pm 0.07$ & $6.47 \%$ & $12.80 \%$ & $5.00 \%$ \\
\hline $\begin{array}{l}\text { South: }(l, b)=\left(0^{\circ},-90^{\circ}\right) \\
\text { Equatorial hemispheres }\end{array}$ & 104 & 1.21 & $1.00 \pm 0.02$ & $0.44 \pm 0.12$ & $(9.11)$ & $(0.02)$ & $(0.20)$ \\
\hline North: $(l, b)=\left(123^{\circ}, 27^{\circ}\right)$ & 136 & 1.32 & $0.99 \pm 0.02$ & $0.34 \pm 0.08$ & $0.2 \%$ & $0.6 \%$ & $64.6 \%$ \\
\hline South: $(l, b)=\left(303^{\circ},-27^{\circ}\right)$ & 104 & 1.18 & $1.04 \pm 0.02$ & $0.35 \pm 0.11$ & $(27.15)$ & $(0.05)$ & $(0.01)$ \\
\hline $\begin{array}{l}\text { Hemispheres max. Asymmetry in } \chi^{2} \text { : } \\
\text { Pole: }(l, b)=\left(120^{\circ}, 25^{\circ}\right) \\
\text { Pole: }(l, b)=\left(300^{\circ},-25^{\circ}\right)\end{array}$ & $\begin{array}{l}133 \\
107\end{array}$ & $\begin{array}{l}1.25 \\
1.24\end{array}$ & $\begin{array}{l}0.98 \pm 0.02 \\
1.04 \pm 0.02\end{array}$ & $\begin{array}{l}0.36 \pm 0.08 \\
0.40 \pm 0.10\end{array}$ & $\begin{array}{c}4.6 \% \\
(35.08)\end{array}$ & $\begin{array}{l}12.2 \% \\
(0.06)\end{array}$ & $\begin{array}{l}93.8 \% \\
(0.04)\end{array}$ \\
\hline $\begin{array}{l}\text { data set B } \\
A_{\mathrm{V}} \leq 1, \sigma_{v}=345 \mathrm{~km} \mathrm{~s}^{-1}, z \leq 0.2 \\
\text { Galactic hemispheres }\end{array}$ & 75 & 0.84 & $1.01 \pm 0.03$ & $-1.42 \pm 1.23$ & & & \\
\hline North: $(l, b)=\left(0^{\circ}, 90^{\circ}\right)$ & 106 & 1.00 & $1.00 \pm 0.03$ & $0.28 \pm 0.08$ & $38.21 \%$ & $9.17 \%$ & $37.98 \%$ \\
\hline $\begin{array}{l}\text { South: }(l, b)=\left(0^{\circ},-90^{\circ}\right) \\
\text { Equatorial hemispheres }\end{array}$ & 72 & 1.28 & $0.97 \pm 0.03$ & $0.34 \pm 0.09$ & $(1.89)$ & $(0.03)$ & $(0.06)$ \\
\hline North: $(l, b)=\left(123^{\circ}, 27^{\circ}\right)$ & 108 & 1.12 & $0.97 \pm 0.03$ & $0.31 \pm 0.08$ & $7.8 \%$ & $4.2 \%$ & $94.2 \%$ \\
\hline $\begin{array}{l}\text { South: }(l, b)=\left(303^{\circ},-27^{\circ}\right) \\
\text { Hemispheres max. Asymmetry in } \chi^{2} \text { : }\end{array}$ & 70 & 1.08 & $1.00 \pm 0.03$ & $0.32 \pm 0.09$ & $(5.86)$ & $(0.03)$ & $(0.01)$ \\
\hline Pole: $(l, b)=\left(235^{\circ}, 15^{\circ}\right)$ & 130 & 1.03 & $1.00 \pm 0.03$ & $0.30 \pm 0.07$ & $62.0 \%$ & $73.3 \%$ & $27.7 \%$ \\
\hline Pole: $(l, b)=\left(55^{\circ},-15^{\circ}\right)$ & 48 & 1.13 & $0.98 \pm 0.03$ & $0.12 \pm 0.11$ & $(13.84)$ & $(0.02)$ & $(0.18)$ \\
\hline
\end{tabular}

the observed effect ( $10 \%$ in distance scale) exceeds our expectations for the effect from peculiar velocities $\left(v /(c \bar{z}) \sim 10^{-3} / \bar{z}\right)$, which in the most extreme case could explain effects at the few per cent level in distance scale (or the SN calibration). However, it is not excluded that large scale bulk motions contribute to the effect, see e.g. Cooray \& Caldwell (2006) who argue that a 5\% variation of $H$ might be possible in a low density bubble. Thus the origin of the anisotropy is most likely either due to a systematic effect in SN search, observation or data analysis or a (large) statistical fluke with a chance below 1:100.

Evidence that we can also exclude the possibility of a statistical fluke comes from the fact that the hemispheres of maximal deviation have poles close to the poles of the equatorial system. This suggests that the origin of the observed anisotropy is, at least partly, due to systematic errors. A possible candidate for a systematic error would be inhomogeneous covering of the North and South sky, but our MC studies reveal that the asymmetry in the number of the objects cannot be held responsible. Exchanging the coordinates of the $\mathrm{SNe}$ (which preserves the asymmetry in number of objects) typically produces skies that are in agreement with the expectation of isotropy. Another possible explanation might be that telescopes or search strategies in the North and South equatorial hemispheres have a systematic calibration off-set.

Yet another hint in favour of an unknown systematic effect is the pattern observed in Figs. 5 and 8. If the Hubble diagrams would be isotropic, we would expect that there are several minima and maxima distributed randomly on the sky. However we observe quite regular patterns in the asymmetry maps. As the asymmetries in data set B are smaller than in data sets A and D, it seems better suited for cosmological analysis. The magnitude of its asymmetries could be consistent with large scale structure
( $1 \%$ effects), but are not statistically significant. However, the reason for the consistency of data set B with isotropy might just be that it does not contain enough $\mathrm{SNe}$. Note that the fit values and their error bars of set B are consistent with the fits of the asymmetric sets A and D.

Putting together all the evidence on the local flow direction in the Universe, mainly based on the analysis of galaxy clusters, Hudson et al. (2004) suggest a bulk flow of $225 \mathrm{~km} \mathrm{~s}^{-1}$ toward $(l, b)=\left(300^{\circ}, 10^{\circ}\right)$ at depth greater than $60 h^{-1} \mathrm{Mpc}$. One would expect that this should be reflected by $\mathrm{SNe}$. However, our maximal asymmetric direction is significantly off-set from the large scale galaxy cluster bulk flow. A closer inspection of Fig. 5 for sets A and D shows a modest, statistically insignificant, asymmetry close to the suggested bulk flow direction.

Our results are also consistent with those from McClure \& Dyer (2007), who find evidence for a significant anisotropy in $H_{0}$ from the HST key project data, but do not claim evidence for an equatorial systematic.

\subsection{Is the local Hubble expansion rate different from the global one?}

We cannot answer that question, as we have no handle in our analysis to the absolute calibration of $\mathrm{SNe}$. If one could exclude that the calibration asymmetries found in this work are due to systematic errors, this would be a strong indication for a variation of the Hubble rate of the order of $10 \%$. If so, the tension between the WMAP 3yr measurment of $H_{0}$ and the determination based on SNe by Sandage et al. (2006) might be reconciled with each other.

The order of magnitude would also be consistent with recent claims by Jha et al. (2006) about the existence of a "Hubble 

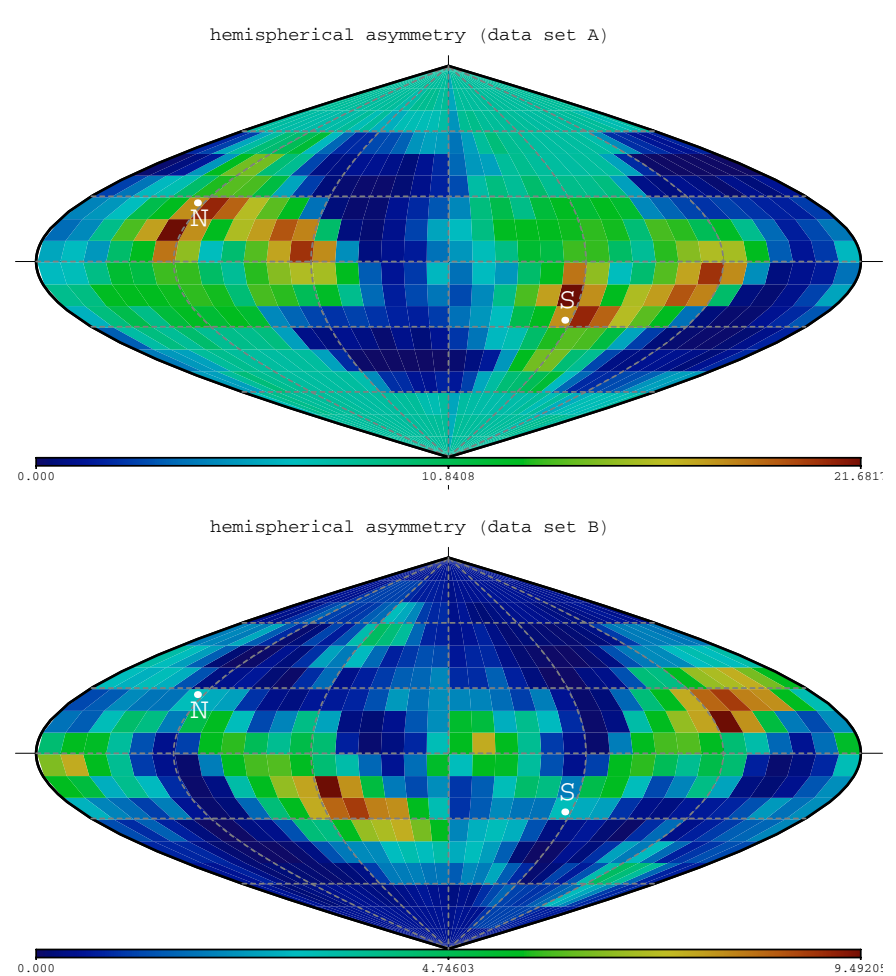

Fig. 8. Hemispherical asymmetry in $\Delta \chi^{2}$ (see also Fig. 5) in the context of the flat $\Lambda \mathrm{CDM}$ model. All SNe with $A_{\mathrm{V}}<1.0$ at any redshift are included in the fits.

bubble"; but more recent analysis (Wang 2007; Conley et al. 2007) does not confirm the claim. Conley et al. (2007) show that the evidence for a "Hubble bubble" depends crucially on how SN colours are modelled. As we think that our test points towards an equatioral North/South systematic, we do not think that this work should be regarded as a support of the idea of a local bubble. However, it might be interesting to check if an equatorial systematic in $\mathrm{SN}$ colours could be found.

\subsection{Is the Universe accelerating today?}

Based on our analysis one cannot answer this question with a straight "yes". The model-independent analysis is fully consistent with an accelerated Universe, but the evidence in favour of acceleration is at most at the $2 \sigma$ level. For the supposedly most accurate compilation of local SNe from Jha et al. (2006) (data set D), we find that $q_{0}=0$ is within the $2 \sigma$ contour (see our Fig. 3). The same is true for all four analysed sets of data (including the SNLS data set, not shown in our Fig. 3). What can be confirmed is that the Einstein-de Sitter model $\left(q_{0}=1 / 2\right)$ is outside the $2 \sigma$ region, and is therefore disfavoured already by the model-independent test at small redshift.

If we assume that the flat $\Lambda C D M$ is the full truth, then the SNe Ia data can indeed provide convincing evidence that the Universe is accelerating. Within that model deceleration $\left(q_{0} \geq 0\right)$ corresponds to $\Omega_{M} \geq 2 / 3$. As can be seen from Fig. 6 , this case is excluded at high confidence. However, we should keep in mind that neither the physics of " $\Lambda$ ", nor that of "CDM" is understood at any depth.

\section{Conclusion}

The purpose of the presented study is to develop tools for and to test the isotropy of Hubble diagrams, which are at the very foundation of modern cosmology. Within our established cosmological model we expect some small deviations from the isotropy, but with the present day accuracy of SNe observations, we would not expect to be able to detect them at high statistical significance (apart from our proper motion). Nevertheless, we set out to apply the hemisphere tests to existing data in order to develop the methodology and to test for systematic effects.

To our surprise we identified a statistically significant asymmetry, which is maximised close to the orientation of the equatorial system. It seems to us that this calls for a thorough investigation of possible systematic effects, which is beyond the scope of this work. Our analysis indicates that there is an off-set in the calibration of SNe between the equatorial hemispheres. Typically SN searches are flux-limited (not red-shift limited) and thus one could imagine that the search in the North and in the South selects samples with different dispersion and mean value in $\mathrm{SN}$ brightness. Our findings would be consistent with a more complete search for nearby SN in the South compared to the North. We think that this study shows, besides the interest in the large scale structure, that a large sky coverage of SN search missions is also important for the issue of systematic errors.

The Hubble diagram is currently the only direct mean to probe the acceleration of the Universe. Most of our evidence for the present day acceleration comes from indirect arguments and relies on a bunch of untested assumptions. Our modelindependent test fails to detect acceleration of the Universe at high statistical significance. It seems to us that it is too early to take accelerated expansion of the Universe for granted, as the evidence heavily relies on the a priori assumption of the $\Lambda \mathrm{CDM}$ model.

Acknowledgements. We thank Camille Bonvin, Craig Copi, Ruth Durrer, Chris Gordon, Steen Hannestad, Stefan Hofmann, Michael Hudson, Dragan Huterer, Ariel Goobar, Martin Kunz, Bruno Leibundgut, Anze Slozar and Glenn Starkman for discussions and comments.

\section{References}

Aldering, G, Goldhaber, G., Perlmutter, S., et al. 1999, ApJ, 517, 565 Bean, R., Doré O., Spergel, D. N., et al. 2006, ApJS, 170, 377 Bonvin, C., Durrer, R., \& Gasparini, M. A. 2006a, Phys. Rev. D, 73, 023523 Bonvin, C., Durrer, R., \& Kunz, M. 2006b, Phys. Rev. Lett., 96, 191302 Conley, A., Carlberg, R. G., Guy, J., et al. 2007, ApJ, 664, L13 Cooray, A., \& Caldwell, R. R. 2006, Phys. Rev. D, 73, 103002 Copi, C. J., Huterer, D., Schwarz, D. J., \& Starkman, G. D. 2007, Phys. Rev. D, 75,023507

Eriksen, H. K., Hansen, F. K., Banday, A. J., Gorski, K. M., \& Lilje, P. B. 2004, ApJ, 605, 14 [Erratum-ibid. 609, 1198]

Filippenko, A. V., Challis, P., Riess, A. G., et al. 1998, AJ, 116, 1009 Germany, L. M. 2001, Ph.D. Thesis, Australian National University Gordon, C., Land, K., \& Slosar, A. 2007, [arXiv: 0705 . 1718] Gupta, S., Saini, T. D., \& Laskar, T. 2007, [arXiv: astro-ph/0701683] Guy, J., Astier, P., Nobili, S., Regnault, N., \& Pain, R. 2005, [arXiv: astro-ph/0506583]

Guy, J., Regnault, N., Astier, P., et al. 2006, A\&A, 447, 31

Halpern, M., Hinshaw, G., Bennett, C. L., et al. 2003, ApJS, 148, 1

Hannestad, S., Thomsen, B., Haugbølle, T., et al. 2006, [arXiv: astro-ph/0612137]

Hannestad, S., Haugbølle, T., \& Thomsen, B., 2007, [arXiv:0705.0979]

Hudson, M. J., Smith, R. J., Lucey, J. R., \& Branchini, E. 2004, MNRAS, 352, 61 
Hui, L., \& Greene, P. B. 2006 Phys. Rev. D, 73, 123526

Jha, S. 2002, Ph.D. Thesis, Harvard University

Jha, S., Riess, A. G., \& Kirshner, R. P. 2006, ApJ, 659, 122

Kolatt, T. S., \& Lahav, O. 2001, MNRAS, 323, 859

Lira, P., Suntzeff, N. B., Phillips, M. M., et al. 1999, AJ, 118, 1766

Madore, B. F., Gibson, B. K., Freedman, W. L., et al. 2001, ApJ, 553, 47

McClure, M. L., \& Dyer, C. C. 2007, New Astron., 12, 533

Miknaitis, G., Stubbs, C. W., Wood-Vasey, M. W., et al.2007

[arXiv: astro-ph/0701041]

Neill, J. D., Hudson, M. J., \& Conley, A., 2007 [arXiv: 0704 . 1654]

Radburn-Smith, D. J., Lucey, J. R., \& Hudson, M. J., 2004, MNRAS, 355, 1378

Riess, A. G., Press, W. H., \& Kirshner, R. P. 1995, ApJ, 445, L91
Riess, A. G., Davis, M., Baker, J., \& Kirshner, R. P. 1997, ApJ, 488, L1

Sandage, A., Tammann, G. A., Saha, A., et al. 2006, ApJ, 653, 843

Sasaki, M., 1987, MNRAS, 228, 653

Schmidt, B. P., Barris, B., Tonry, J. L., et al. 2003, ApJ, 594, 1

Strolger, L.-G., Tonry, J., Riess, A. G., et al. 2004, ApJ, 607, 665

Strolger, L.-G., Casertano, S., Riess, A. G., et al. 2006 [arXiv:astro-ph/0611572]

Tonry, J., Bolondin, S., Barris, B. J., et al. 2004, ApJ, 602, 571

Wang, L. 2007 [arXiv: 0705 .0368]

Weinhorst, B. 2006, Anisotropien von Hubble-Diagrammen, diploma thesis, University of Bielefeld; URL:

www.physik.uni-bielefeld.de/cosmology/theses.html

Zehavi, I., Riess, A. G., Kirshner, R. P. \& Dekel, A. 1998, ApJ, 503, 483 